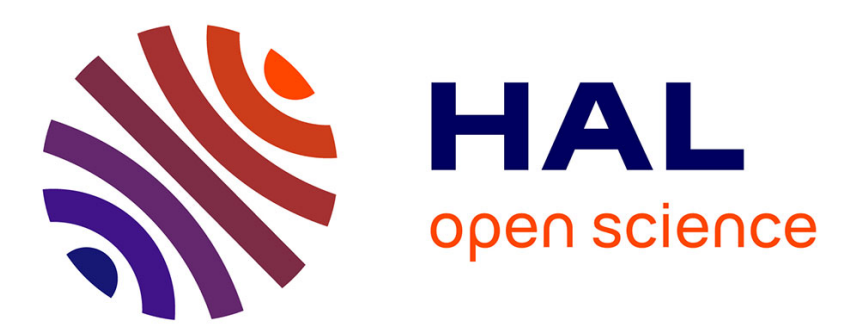

\title{
Modeling of thin isotropic elastic plates with small piezoelectric inclusions and distributed electric circuits
}

\author{
Eric Canon, Michel Lenczner
}

\section{To cite this version:}

Eric Canon, Michel Lenczner. Modeling of thin isotropic elastic plates with small piezoelectric inclusions and distributed electric circuits. Mathematical Methods in the Applied Sciences, 2015, 38 (1), pp.66 - 86. hal-02963431

\section{HAL Id: hal-02963431 \\ https://hal.science/hal-02963431}

Submitted on 10 Oct 2020

HAL is a multi-disciplinary open access archive for the deposit and dissemination of scientific research documents, whether they are published or not. The documents may come from teaching and research institutions in France or abroad, or from public or private research centers.
L'archive ouverte pluridisciplinaire HAL, est destinée au dépôt et à la diffusion de documents scientifiques de niveau recherche, publiés ou non, émanant des établissements d'enseignement et de recherche français ou étrangers, des laboratoires publics ou privés. 


\title{
Modelling of thin isotropic elastic plates with small piezoelectric inclusions and distributed electric circuits. Models for inclusions larger or comparable to the thickness of the plate.
}

\author{
Éric Canon \\ Pres université de Lyon, \\ UMR CNRS 5208, \\ Université Jean Monnet de Saint-Étienne, \\ Institut Camille Jordan, \\ 23 rue du docteur Paul Michelon, F-42 023 Saint-Étienne. France. \\ email: eric.canon@univ-st-etienne.fr \\ Michel Lenczner \\ FEMTO-ST, Département Temps-Fréquence, \\ University of Franche-Comté, \\ 26 chemin de l'Epitaphe, 25030 Besançon Cedex, France \\ michel.lenczner@utbm.fr
}

November 4, 2013

\begin{abstract}
This paper is the second part of a work devoted to the modelling of thin elastic plates with small, periodically distributed piezoelectric inclusions. We consider the equations of linear elasticity coupled with the electrostatic equation, with various kinds of electric boundary conditions. We derive the corresponding effective models when the thickness $a$ of the plate and the characteristic dimension $\varepsilon$ of the inclusions tend together to zero, in the two following situations: first when $a \simeq \varepsilon$, second when $a / \varepsilon$ tends to zero.
\end{abstract}

Mathematics Subject Classifications (1991): 35B27 Homogenization of partial differential equations in media with periodic structures, 73B27 nonhomogeneous materials and homogenization.

Key words: linear elasticity, piezoelectricity, homogenization, plate theory, composite materials, prescribed electric potential, local electric circuits, nonlocal electric circuits, transfinite networks, smart materials. 


\section{Introduction}

1.1 General. This paper is the third and last part of a systematic work devoted to the derivation of effective models for thin piezoelectric/elastic composite plates including elementary electric circuits connected to the upper and lower faces of piezoelectric transducers. It is motivated by an important development of piezocomposites used for instance for distributed control in vibroacoustics $[8,11,12,25]$ or as sensors in phased arrays. In [5], we considered three-dimensional elastic plates with a small number of piezoelectric inclusions and derived effective models when the thickness $a$ of the plate tends to zero. In [7], effective models of thin plates with a large number of $\varepsilon$-periodically distributed piezoelectric inclusions have been obtained in the case $\varepsilon<<a$ by letting $a$, $\varepsilon$ and $\varepsilon / a$ simultaneously tend to zero. The aim of the present paper is to consider the two other possible asymptotic cases, namely $a / \varepsilon \rightarrow 0$ and $\varepsilon / a \rightarrow 1$. We note that the models for $a / \varepsilon \rightarrow 0$ were already presented in the note $[6]$.

As in $[5,7]$, different kinds of boundary conditions are considered on the metallized upper faces of the inclusions, corresponding to different possible types of control: prescribed electric potential (or Dirichlet conditions) if the tension is controlled, prescribed electric displacement field (or Neumann conditions) if the current is controlled, local and nonlocal mixed conditions if the inclusions are connected to R-L-C circuits (the nonlocal conditions corresponding to inclusions that are connected to each other via R-L-C circuits).

Following a principle used in [5, 7], the derivation of the models is made in the space of gradients of solutions. This leads to a more synthetic and readable presentation of the results. We combine the two-scale convergence $[1,19]$ for homogenization and classical arguments of thin plates theory $[9,10,20]$. Let us quote the pioneer work of Caillerie [4], who considered the case of thin static elastic plates with periodic coefficients, using the Tartar's method of oscillating test functions [2, 22]. However, in [4] the parameters $a$ and $\varepsilon$ tend successively and independently to zero, except for $a \simeq \varepsilon$.

Despite their relative formal complexity, the effective models have a rather simple structure. In the case $a / \varepsilon \rightarrow 0$, for Dirichlet, and local mixed conditions (from a mathematical point of view the prescribed electric displacement field conditions happen to be a special case of mixed conditions and is not treated separately), the limit model has the same form as the elastic plate model, the influence of piezoelectric inclusion only appears in the definition of the effective coefficients and as a source term. For nonlocal mixed conditions, a coupling arises between mechanical effects and the transverse component of the electric field, because of a Laplace operator in the in-plane direction induced by the electric circuits. The resulting operator is a special case of those encountered in the homogenization of periodic electric circuits [15-17] or in transfinite networks [27, 28], the later being analyzed from a different point of view. We also quote [21] where a wide variety of in-plane operators are generated by a periodic network of resistances. In the case $a / \varepsilon \rightarrow 1$, for Dirichlet conditions the limit model also has the same form as the purely elastic thin plate model, but a coupling arises even in the case of local mixed conditions.

Concerning thin piezoelectric structures, let us also mention: Ghergu and al [14] who 
consider perforated piezoelectric shells with fixed thickness; Licht and Weller [18, 26]; Sene [23] and Figueiredo and Leal [13] who consider piezoelectric plates without homogenization. Remark however that in [13] general anisotropic models are considered. Finally, let us remark that the models used in vibro-acoustic applications, as in [8, 11, 12, 25], are often based on Bloch wave decompositions which seems a priori not compatible with the homogenization method used in this paper. However, the homogenized model for the wave equation in [3] builds a bridge between the two views and constitutes a perspective for further works.

1.2 Detailed contents. Section 2 is devoted to the setting of the initial 3-dimensional equations of static linearized elasticity and piezoelectricity. The piezoelectric inclusions are assumed to be strictly included in an insulating elastic matrix.

As our work is more about introducing piezoelectric plates with elementary electric devices and about mixing homogenization and plates theory, for simplicity and efficiency, following [4], we assume that the material coefficients are constant in the thickness direction. This is usually the case in applications, as the matrix and the piezoelectric ceramics are homogeneous materials. In the same spirit, the upper and lower faces of the piezoelectric inclusions are assumed to be metallized, that is to be covered with a thin conductive film. However, from the mathematical point of view, it might be interesting to obtain more general models by removing these technical assumptions and considering fully nonhomogeneous materials as in [13], or multi-layered plate by adapting to the present work to formalism proposed in [5].

The mechanical boundary conditions applied to the plate are prescribed surface forces on its lower and upper faces and on part of its lateral boundary, and prescribed mechanical displacement on the remaining part. For the Maxwell-Gauss equations, we consider prescribed electrical potential on the lower faces of the inclusions (in practice these faces are connected to ground and the electric potential is zero). As already mentioned in Section 1.1 various boundary conditions are considered on the upper faces of the inclusions, corresponding to connections to electric or electronic devices. These conditions are detailed in section 2.4. Some of them are, to our knowledge, unusual in plates theory, and thus, constitute one of the interesting point of our work.

The weak formulations of the system are stated in Section 3. For a concise formulation covering all kinds of boundary conditions, we adopt synthetic tensorial notations rather than fully extended formulae. We strongly believe that this allows a better legibility of computations as well as of limit models.

The precise assumptions on the data are presented in Section 4. In particular, we give the correct scalings, or, from a more concrete point of view, how electrical circuits have to be chosen to obtain a significant influence on the effective behaviour of the material. Resulting a priori estimates and first convergence results are given in Sections 4.2 and 4.3 .

Sections 5 and 6 are devoted to the statement of the main results i.e. the effective twodimensional plate model for each type of electrical boundary condition in the case where 
the plate thickness is much smaller than the inclusions size (Theorem 5.1, Section 5) and in the case where these two parameters are of the same order (Theorem 6.1, Section 6).

Theorem 5.1 is proved in Section 7. The proof is in three steps. The first one, which is mathematically the most difficult consists in characterizing the two-scale limits of the strain and of the electrical field. These results are new, even in the case of pure elasticity.In Caillerie [4] - as two-scale convergence did not exist at the time - only weak limits were considered. The second step consists in eliminating the microscopic variable by computing the microscopic fields in terms of the macroscopic fields. We use here the classical arguments of linear homogenization. The third step consists in eliminating the transverse components, or part of the transverse components of the fields (according to the model), that are computed with respect to the other ones. This elimination slightly departs from the classical plates theory, because of the non standard boundary conditions on the upper and lower faces of the inclusions that are considered in the present paper.

Theorem 6.1 is proved in Section 8. As here $a \simeq \varepsilon$ is assumed, the proof is only in two steps. First, characterization of the limit, second, simultaneous elimination of the local and of (part of) the transverse components.

We use the same formalism as in [5,7], based on tensorial notations and products, and on simple algebraic operations such as projections. It allows to deal relatively easily with complex computations. Completely explicit formulae would require a lot of room, to the detriment of legibility. Step 2 and 3 of our proofs are almost formal computation and are easily adapted from one variant to the other. A coupling with the formalism introduced in [5] for multilayered plates models is easily conceivable.

\section{Equations of 3-dimensional piezoelectricity}

2.1 Geometry. The three-dimensional plate with thickness $a>0$ is represented by $\left.\Omega^{a}=\omega \times\right]-a, a\left[, \omega\right.$ being a bounded domain of $\mathbb{R}^{2}$, see Figure 1 . Using the change of scales and variables introduced in [10], we shall work on the fixed domain $\Omega=\omega \times]-1,1[$. The domain $\omega$ is divided into two subdomains $\omega_{1}^{\varepsilon}$ and $\omega_{2}^{\varepsilon}$ that are constructed as follows. Let $Y=]-1 / 2,1 / 2\left[{ }^{2}\right.$ and $Y_{1}$ be a strict smooth subdomain of $Y$, see Figure 2, let $\mathbb{I}^{\varepsilon}$ be the set of multi-index $\mathbf{i}=\left(i_{1}, i_{2}\right) \in \mathbb{Z}^{2}$ such that $\varepsilon\left(\mathbf{i}+Y_{1}\right)$ is strictly included in $\omega$; then $\omega_{1}^{\varepsilon}=\bigcup_{\mathbf{i} \in \mathbb{I}^{\varepsilon}} \varepsilon\left(\mathbf{i}+Y_{1}\right)$ while $\omega_{2}^{\varepsilon}=\omega \backslash \bar{\omega}_{1}^{\varepsilon}$. Let $b=(a, \varepsilon)$; the set $\left.\Omega_{2}^{b}=\omega_{2}^{\varepsilon} \times\right]-a, a[$ represents the elastic matrix of the body, while $\left.\Omega_{1}^{b}=\omega_{1}^{\varepsilon} \times\right]-a, a[$ is the set of all piezoelectric inclusions. The boundary of $\omega$ is divided into two regular parts $\gamma_{D}$ and $\gamma_{N}$, with $\left|\gamma_{D}\right|>0$. The boundary of $\Omega^{a}$ is thereby divided into $\left.\Gamma_{D}^{a}=\gamma_{D} \times\right]-a, a\left[\right.$ and $\Gamma_{N}^{a}=\left(\gamma_{N} \times\right]-a, a[) \cup$ $(\omega \times\{-a, a\})$. The boundary of $\Omega_{1}^{b}$ is divided into $\Gamma_{1}^{b+}=\omega_{1}^{\varepsilon} \times\{a\}, \Gamma_{1}^{b-}=\omega_{1}^{\varepsilon} \times\{-a\}$ and $\left.\Gamma_{1}^{b}=\partial \omega_{1}^{\varepsilon} \times\right]-a, a\left[\right.$. The outer unit normals to the boundaries of $\Omega^{a}$ and $Y$ are denoted by $\mathbf{n}$ and $\mathbf{n}_{Y}$, respectively.

For any inclusion $\left.\varepsilon\left(\mathbf{i}+Y_{1}\right) \times\right]-a, a\left[\right.$ such that $\mathbf{i} \in \mathbb{I}^{\varepsilon}$, the mean value on the upper face $\varepsilon\left(\mathbf{i}+Y_{1}\right) \times\{a\}$ is denoted by $<.>_{\mathbf{i}}$. For any function $\psi$ on $\Omega^{a}, \psi_{\mathbf{i}}$ designates its restriction to the inclusion $\left.\varepsilon\left(\mathbf{i}+Y_{1}\right) \times\right]-a, a[$. 


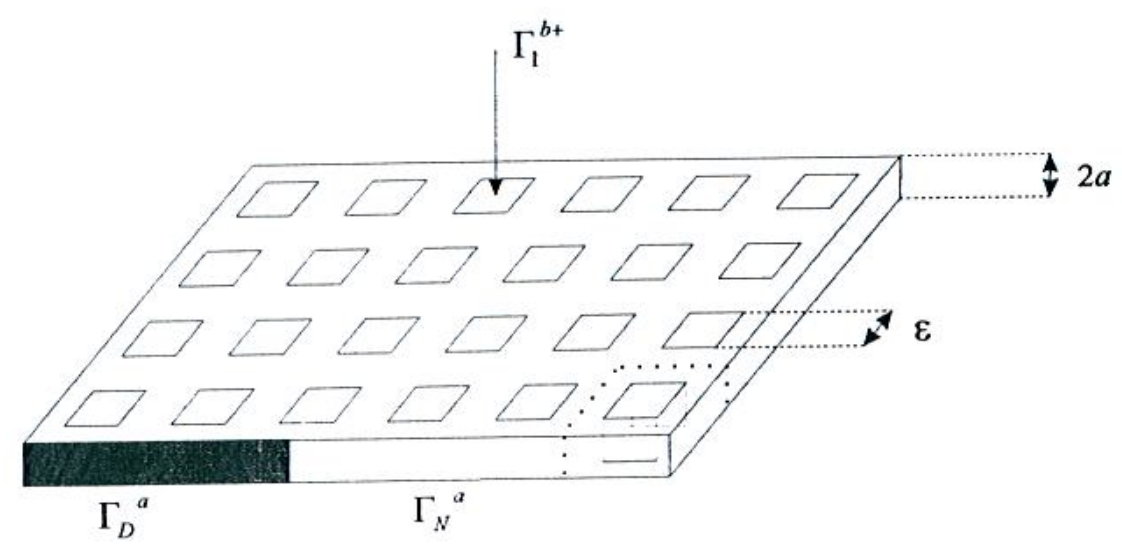

Figure 1: Composite plate with piezoelectric inclusions.

The space variables are $x^{a}=\left(\hat{x}, x_{3}^{a}\right) \in \Omega^{a}$ where $\left.x_{3}^{a} \in\right]-a, a\left[, \hat{x}=\left(x_{1}, x_{2}\right) \in \omega\right.$ and $y=\left(y_{1}, y_{2}\right) \in Y$. The derivatives with respect to $x_{\alpha}, x_{3}^{a}$ and $y_{\alpha}$ are denoted by $\partial_{\alpha}, \partial_{3}$ and $\partial_{y_{\alpha}}$, respectively.

When referring to the fixed domain $\Omega$, the geometric notation is the same, the subscript $a$ being removed if necessary.

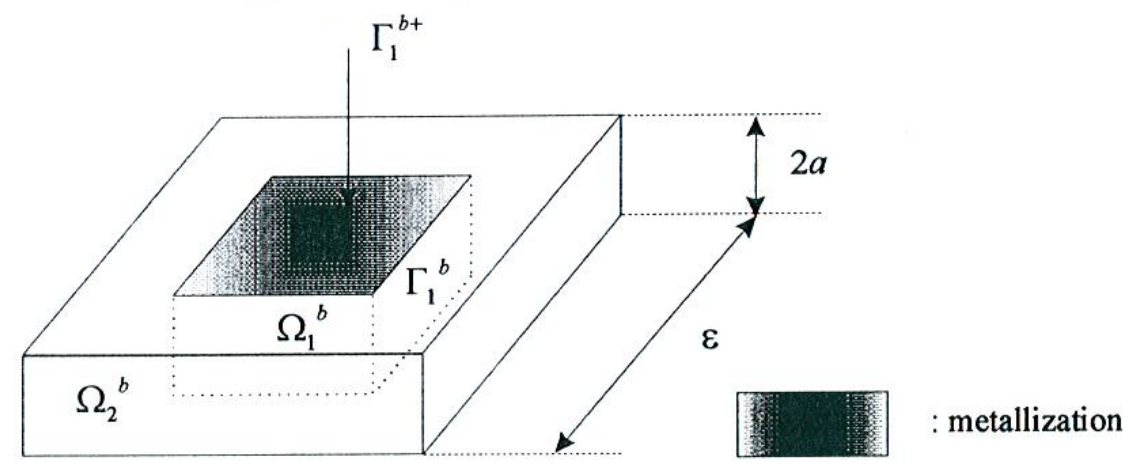

Figure 2: Elementary cell, with piezoelectric inclusion and metallization.

2.2 Other notations. Bold characters are used for vector and matrix valued functions and for the corresponding functional spaces. We constantly use Einstein's convention of summation on repeated indices with the additional convention that latin and greek indices are varying from 1 to 3 and from 1 to 2 respectively. Throughout the paper $c$ and $C$ designate generic positive constants, not depending on $a$ and $\varepsilon$. 
Also:

$$
\text { inutile }
$$

Last, vector and vectorial notations are a priori meant in line. When a given vector $\mathbf{V}$ is meant in column, we write: ${ }^{t} \mathbf{V}$. For instance for

$$
\mathbf{V}=\left(\left(s_{\alpha \beta}(\mathbf{v})\right)_{\alpha, \beta=1,2}, \quad\left(a^{-1} s_{\alpha 3}(\mathbf{v})\right)_{\alpha=1,2}, a^{-2} s_{33}(\mathbf{v})\right),
$$

with ${ }^{t} \mathbf{V}$, we mean

$$
{ }^{t} \mathbf{V}=\left(\begin{array}{l}
\left(s_{\alpha \beta}(v)\right)_{\alpha, \beta=1,2} \\
\left(a^{-2} s_{\alpha 3}(v)\right)_{\alpha=1,2} \\
a^{-2} s_{33}(v)
\end{array}\right)
$$

Note that this convention leads us to writings that slightly differ from the one in $[6,7]$, but it seems more coherent to us now.

2.3 Equations of 3-dimensional piezoelectricity. The mechanical displacements $\mathbf{u}^{b}=\left(u_{i}^{b}\right)_{i=1,2,3}$ and the electric potential $\varphi^{b}$ are governed by the linearized equations of piezoelectricity in their static version,

$$
\left\{\begin{array}{l}
-\partial_{j} \sigma_{i j}^{b}=f_{i}^{b} \text { in } \Omega^{a}, \quad \sigma_{i j}^{b} n_{j}=g_{i}^{b} \text { on } \Gamma_{N}^{a}, u_{i}^{b}=0 \text { on } \Gamma_{D}^{a} \\
-\partial_{j} D_{j}^{b}=0 \text { in } \Omega_{1}^{b}, D_{j}^{b} \cdot n_{i}=0 \text { on } \Gamma_{1}^{b}
\end{array}\right.
$$

for $\mathrm{i}=1,2,3$, where

$$
\begin{gathered}
\left\{\begin{array}{l}
\sigma_{i j}^{b}=R_{i j k l}^{\varepsilon} s_{k l}\left(\mathbf{u}^{b}\right)+d_{k i j}^{\varepsilon} \partial_{k} \varphi^{b}, \\
D_{j}^{b}=-d_{j k l}^{\varepsilon} s_{k l}\left(\mathbf{u}^{b}\right)+c_{j k}^{\varepsilon} \partial_{k} \varphi^{b},
\end{array}\right. \\
\forall \mathbf{v} \in \mathbf{H}^{1}\left(\Omega^{a}\right), \quad s_{i j}(\mathbf{v})=\frac{1}{2}\left(\partial_{i} v_{j}+\partial_{j} v_{i}\right) .
\end{gathered}
$$

The assumptions on the volume and surface forces $\mathbf{f}^{b}:=\left(f_{i}^{b}\right)_{i=1,2,3}$ and $\mathbf{g}^{b}:=\left(g_{i}^{b}\right)_{i=1,2,3}$ are specified below, in Section 4.1. The stiffness tensor $\mathbf{R}^{\varepsilon}:=\left(R_{i j k l}^{\varepsilon}\right)_{i, j, k, l=1,2,3}$, the piezoelectricity tensor $\mathbf{d}^{\varepsilon}:=\left(d_{k i j}^{\varepsilon}\right)_{i, j, k=1,2,3}$ and the permitivity tensor $\mathbf{c}^{\varepsilon}:=\left(c_{i j}^{\varepsilon}\right)_{i, j=1,2,3}$ are assume to satisfy the symmetry conditions:

$$
\forall i, j, k, l \in\{1,2,3\}, \quad R_{i j k l}^{\varepsilon}=R_{k l i j}^{\varepsilon}=R_{j i k l}^{\varepsilon}, \quad c_{i j}^{\varepsilon}=c_{j i}^{\varepsilon}, \quad d_{k i j}^{\varepsilon}=d_{k j i}^{\varepsilon} .
$$

Note that, as we assume that the inclusions are electrically insulated from the elastic matrix, no Gauss-Maxwell equation is needed in $\Omega_{2}^{b}$. However, we let for convenience

$$
\mathbf{c}^{\varepsilon}=\mathbf{0}, \quad \mathbf{d}^{\varepsilon}=\mathbf{0} \quad \text { in } \Omega_{2}^{b} .
$$

We go now into detail about the different kinds of electric boundary conditions that are considered in this paper.

2.4 Electric boundary conditions on $\Gamma_{1}^{b+} \cup \Gamma_{1}^{b-}$. The four kinds of electric boundary conditions are summarized here. Further explanations and comments can be found in [7]. 
(i) Prescribed electric potential (Dirichlet conditions):

$$
\varphi^{b}=\varphi_{m}^{b}+a \varphi_{c}^{b} \text { on } \Gamma_{1}^{b+}, \quad \varphi^{b}=\varphi_{m}^{b}-a \varphi_{c}^{b} \text { on } \Gamma_{1}^{b-},
$$

$\varphi_{m}^{b}$ and $\varphi_{c}^{b}$ being two given functions on $\omega_{1}^{\varepsilon}$.

(ii) Prescribed electric displacement field (Neumann conditions), local and nonlocal electric circuits (local and nonlocal mixed conditions):

Let us introduce the shift operators defined from $\mathbb{I}^{\varepsilon}$ to $\mathbb{Z}^{2}$ by

$$
\left\{\begin{array}{l}
T_{+1}^{1}: \mathbf{i} \mapsto\left(i_{1}+1, i_{2}\right), T_{+1}^{2}: \mathbf{i} \mapsto\left(i_{1}, i_{2}+1\right), \\
T_{-1}^{1}: \mathbf{i} \mapsto\left(i_{1}-1, i_{2}\right), T_{-1}^{2}: \mathbf{i} \mapsto\left(i_{1}, i_{2}-1\right) .
\end{array}\right.
$$

Let $\varphi_{m}^{b}$ be a given function on $\omega_{1}^{\varepsilon}$. For all $\mathbf{i} \in \mathbb{I}^{\varepsilon}$, we pose

$$
\bar{\varphi}_{\mathbf{i}}^{b}=\varphi_{\mathbf{i}}^{b}-\varphi_{m, \mathbf{i}}^{b}
$$

The boundary conditions for the electrostatic equation $(2)_{2}$ on $\Gamma_{1}^{b+} \cup \Gamma_{1}^{b-}$ are then

$$
\left\{\begin{array}{l}
\forall \mathbf{i} \in \mathbb{I}^{\varepsilon}, \quad<\mathbf{D}^{b} . \mathbf{n}>_{\mathbf{i}}=\frac{G_{1}}{a \varepsilon^{2}} \sum_{\alpha=1}^{2}\left(\bar{\varphi}_{T_{-1}^{\alpha}(\mathbf{i})}^{b}-2 \bar{\varphi}_{\mathbf{i}}^{b}+\bar{\varphi}_{T_{+1}^{\alpha}(\mathbf{i})}^{b}\right)-\frac{G}{a} \bar{\varphi}_{\mathbf{i}}^{b}+h^{b} \quad \text { on } \Gamma_{1}^{b+}, \\
\varphi^{b}=\varphi_{m}^{b} \quad \text { on } \Gamma_{1}^{b-} .
\end{array}\right.
$$

The finite difference operator on $\bar{\varphi}_{\mathbf{i}}^{b}$ is completed by the analog of a discrete Neumann boundary condition to the free ends of the circuit

$$
\bar{\varphi}_{T_{-1}^{\alpha}(\mathbf{i})}^{b}-\bar{\varphi}_{\mathbf{i}}^{b}=0 \quad \text { if } T_{-1}^{\alpha}(\mathbf{i}) \notin \mathbb{I}^{\varepsilon}, \quad \bar{\varphi}_{T_{+1}^{\alpha}(\mathbf{i})}^{b}-\bar{\varphi}_{\mathbf{i}}^{b}=0 \quad \text { if } T_{+1}^{\alpha}(\mathbf{i}) \notin \mathbb{I}^{\varepsilon}
$$

which also means that the current is vanishing in the corresponding branches.

In (8), $G_{1}$ and $G$ are given nonnegative constants. If $G=G_{1}=0$ the conditions on $\Gamma_{1}^{b+}$ are Neumann condition (prescribed electric displacement field). If $G>0$ and $G_{1}=0$ those are local mixed conditions. If $G G_{1}>0$ those are nonlocal mixed conditions. If $G_{1}=0$, the condition (9) is of course not relevant.

Although from a physical point of view it has its own meaning, the case of Neumann conditions does not need to be treated separately; one just have to let $G=0$ in the effective models obtained in Theorem 5.1 and Theorem 6.1 for local mixed conditions. In the situation $a / \varepsilon \rightarrow 0$, local and nonlocal conditions lead to different developments and limit models.

As we assume all the faces to be metallized, the functions $\bar{\varphi}^{b}, \varphi_{m}^{b}$ and $\bar{\varphi}_{c}^{b}$ are constant on each metallized face of inclusions and assuming that the current is provided by a single wire, the same holds true for $h^{b}$.

Finally, in order to use, as much as possible, common formulations for the different boundary conditions, we define $h^{b}, \varphi_{c}^{b}, G$ and $G_{1}$ in all cases, with the conventions that

$$
h^{b}=0 \text { and } G=G_{1}=0 \text { for Dirichlet conditions, }
$$


and

$$
\varphi_{c}^{b}=0 \text { for mixed conditions. }
$$

\section{Weak formulations}

The aim of the present section is the statement of the weak formulation of the equations of Section 2, on the fixed domain $\Omega$. We use the standard change of variables $x^{a} \rightarrow x=\left(x_{1}, x_{2}, x_{3}^{a} / a\right)$ and the appropriate scaling for volume forces, surface forces and displacements fields, see [10] and also [9],

$$
\left\{\begin{array}{l}
\forall x \in \Omega, \quad \hat{\mathbf{u}}^{b}(x)=\left(u_{1}^{b}\left(x^{a}\right), u_{2}^{b}\left(x^{a}\right), a u_{3}^{b}\left(x^{a}\right)\right), \\
\forall x \in \Omega, \hat{\mathbf{f}}^{b}(x)=\left(f_{1}^{b}\left(x^{a}\right), f_{2}^{b}\left(x^{a}\right), a^{-1} f_{3}^{b}\left(x^{a}\right)\right), \\
\left.\forall x \in \gamma_{N} \times\right]-1,1\left[, \quad \hat{\mathbf{g}}^{b}(x)=\left(g_{1}^{b}\left(x^{a}\right), g_{2}^{b}\left(x^{a}\right), a^{-1} g_{3}^{b}\left(x^{a}\right)\right)\right. \\
\forall x \in \omega \times\{-1,1\}, \quad \hat{\mathbf{g}}^{b}(x)=a^{-1}\left(g_{1}^{b}\left(x^{a}\right), g_{2}^{b}\left(x^{a}\right), a^{-1} g_{3}^{b}\left(x^{a}\right)\right) .
\end{array}\right.
$$

The current source $h^{b}$, the electric potential $\varphi^{b}, \varphi_{m}^{b}$ and $\varphi_{c}^{b}$ are left unchanged. As in the sequel, we only work on the reference domain $\Omega$, no confusion might occur, so for simplicity we keep the notation $\mathbf{u}^{b}, \mathbf{f}^{b}, \mathbf{g}^{b}, h^{b}, \varphi^{b}, \varphi_{m}^{b}$ and $\varphi_{c}^{b}$, without hats.

For any $\mathbf{V}=(\mathbf{v}, \psi) \in \mathbf{H}^{1}(\Omega) \times H^{1}\left(\Omega_{1}^{\varepsilon}\right)$, we let

$$
\left\{\begin{array}{l}
\left.\mathbf{K}^{a}(\mathbf{v})=\left(K_{\alpha \beta}^{a}(\mathbf{v}), K_{\alpha 3}^{a}(\mathbf{v})\right), K_{33}^{a}(\mathbf{v})\right)=\left(s_{\alpha \beta}(\mathbf{v}), a^{-1} s_{\alpha 3}(\mathbf{v}), a^{-2} s_{33}(\mathbf{v})\right) \\
\mathbf{L}^{a}(\psi)=\left(L_{\alpha}^{a}(\psi), L_{3}^{a}(\psi)\right)=\left(\partial_{\alpha} \psi, a^{-1} \partial_{3} \psi\right) \\
\mathbf{M}^{a}(\mathbf{V})=\left(\mathbf{K}^{a}(\mathbf{v}), \mathbf{L}^{a}(\psi)\right)
\end{array}\right.
$$

We put together the tensors $\mathbf{R}^{\varepsilon}, \mathbf{d}^{\varepsilon}$ and $\mathbf{c}^{\varepsilon}$ in a global stiffness-piezoelectricity-permitivity tensor $\mathcal{R}^{\varepsilon}$, which is the $10 \times 10$ symmetric matrix written in a format compatible with (12):

$$
\mathcal{R}^{\varepsilon}=\left(\begin{array}{ccccc}
R_{\alpha \beta \gamma \delta}^{\varepsilon} & 2 R_{\alpha \beta \gamma 3}^{\varepsilon} & R_{\alpha \beta 33}^{\varepsilon} & d_{\gamma \alpha \beta}^{\varepsilon} & d_{3 \alpha \beta}^{\varepsilon} \\
2 R_{\alpha 3 \gamma \delta}^{\varepsilon} & 4 R_{\alpha 3 \gamma 3}^{\varepsilon} & 2 R_{\alpha 333}^{\varepsilon} & 2 d_{\gamma \alpha 3}^{\varepsilon} & 2 d_{3 \alpha 3}^{\varepsilon} \\
R_{33 \gamma \delta}^{\varepsilon} & 2 R_{33 \gamma 3}^{\varepsilon} & R_{3333}^{\varepsilon} & d_{\gamma 33}^{\varepsilon} & d_{333}^{\varepsilon} \\
-d_{\alpha \gamma \delta}^{\varepsilon} & -2 d_{\alpha \gamma 3}^{\varepsilon} & -d_{\alpha 33}^{\varepsilon} & c_{\alpha \gamma}^{\varepsilon} & c_{\alpha 3}^{\varepsilon} \\
-d_{3 \gamma \delta}^{\varepsilon} & -2 d_{3 \gamma 3}^{\varepsilon} & -d_{333}^{\varepsilon} & c_{3 \gamma}^{\varepsilon} & c_{33}^{\varepsilon}
\end{array}\right)
$$

The linear forms associated with the mechanical load and the electrical current source are

$$
\ell_{u}^{b}(\mathbf{v})=\int_{\Omega} f_{i}^{b} v_{i} \mathrm{~d} x+\int_{\Gamma_{N}} g_{i}^{b} v_{i} \mathrm{~d} s, \text { and } \ell_{\varphi}^{b}\left(\tilde{L}_{3}\right)=\int_{\Omega_{1}^{\varepsilon}} h^{b} \tilde{L}_{3} \mathrm{~d} x .
$$

Note that $h^{b}$ is a priori defined on $\Gamma_{1}^{\varepsilon+}$ (or equivalently on $\omega_{1}^{\varepsilon}$ ) but is trivially extended to a function on $\Omega_{1}^{\varepsilon}$ which does not depend on $x_{3}$. Given the assumption on metallization, the set of admissible electric potential is

$$
H_{c}^{1}\left(\Omega_{1}^{\varepsilon}\right)=\left\{\psi \in H^{1}\left(\Omega_{1}^{\varepsilon}\right) ; \psi \text { is constant on each connected part of } \Gamma_{1}^{\varepsilon+} \cup \Gamma_{1}^{\varepsilon-}\right\} .
$$


The relevant functional space is then specific to each electric boundary condition.

(i) Dirichlet conditions:

$$
\left\{\begin{array}{c}
\mathbf{W}_{D}^{b}=\mathbf{W}^{\varepsilon}\left(\varphi_{m}^{b}, \varphi_{c}^{b}\right)=\left\{(\mathbf{v}, \varphi) \in \mathbf{H}^{1}(\Omega) \times H_{c}^{1}\left(\Omega_{1}^{\varepsilon}\right) ; \mathbf{v}=\mathbf{0} \text { on } \Gamma_{D}\right. \\
\left.\varphi=\varphi_{m}^{b}+a \varphi_{c}^{b} \text { on } \Gamma_{1}^{\varepsilon+}, \varphi=\varphi_{m}^{b}-a \varphi_{c}^{b} \text { on } \Gamma_{1}^{\varepsilon-}\right\} \\
\mathbf{W}^{\varepsilon}=\mathbf{W}^{\varepsilon}(0,0) .
\end{array}\right.
$$

(ii) Mixed conditions:

$$
\left\{\begin{array}{l}
\mathbf{W}_{D}^{b}=\mathbf{W}^{\varepsilon}\left(\varphi_{m}^{b}\right)=\left\{(\mathbf{v}, \varphi) \in \mathbf{H}^{1}(\Omega) \times H_{c}^{1}\left(\Omega_{1}^{\varepsilon}\right) ; \mathbf{v}=\mathbf{0} \text { on } \Gamma_{D}, \varphi=\varphi_{m}^{b} \text { on } \Gamma_{1}^{\varepsilon-}\right\}, \\
\mathbf{W}^{\varepsilon}=\mathbf{W}^{\varepsilon}(0) .
\end{array}\right.
$$

The backward difference operator $\nabla_{\hat{x}}^{\varepsilon}$ is defined inclusion by inclusion by

$$
\forall \mathbf{i} \in \mathbb{I}^{\varepsilon}, \forall \psi \in H_{c}^{1}\left(\Omega_{1}^{\varepsilon}\right), \quad\left(\nabla_{\hat{x}}^{\varepsilon} \psi\right)_{\mathbf{i}}=\varepsilon^{-1}\left(\psi_{\mathbf{i}}-\psi_{T_{-1}^{1}(\mathbf{i})}, \psi_{\mathbf{i}}-\psi_{T_{-1}^{2}(\mathbf{i})}\right) .
$$

Letting $\mathbf{U}^{b}=\left(\mathbf{u}^{b}, \varphi^{b}\right)$, with the conventions (6), (10) and (11), the weak formulations on the scaled domain $\Omega$ for the coupled problems (2)-(7)-(8), are summarized by:

$$
\left\{\begin{array}{l}
\mathbf{U}^{b}=\left(\mathbf{u}^{b}, \varphi^{b}\right) \in \mathbf{W}_{D}^{b}, \quad \text { and for all } \mathbf{V}=(\mathbf{v}, \psi) \in \mathbf{W}^{\varepsilon} \\
\int_{\Omega} \mathbf{M}^{a}(\mathbf{V}) \mathcal{R}^{\varepsilon}{ }^{t} \mathbf{M}^{a}\left(\mathbf{U}^{b}\right) \mathrm{d} x+2 \int_{\Omega_{1}^{\varepsilon}} G \mathcal{M}\left(L_{3}^{a}\left(\varphi^{b}\right)\right) \mathcal{M}\left(L_{3}^{a}(\psi)\right) \mathrm{d} x \\
\quad+2 \int_{\Omega_{1}^{\varepsilon}} G_{1} \nabla_{\hat{x}}^{\varepsilon} \mathcal{M}\left(L_{3}^{a}\left(\varphi^{b}\right)\right) \cdot \nabla_{\hat{x}}^{\varepsilon} \mathcal{M}\left(L_{3}^{a}(\psi)\right) \mathrm{d} x=\ell_{u}^{b}(\mathbf{v})+\ell_{\varphi}^{b}\left(L_{3}^{a}(\psi)\right) .
\end{array}\right.
$$

\section{Assumptions on the data - A priori estimates - Convergences}

4.1 Two-scale convergence. As two-scale convergence is an important tool of the paper, before stating the assumptions on the data and the first convergence results, that are expressed in terms of two-scale convergence, let us recall some basic facts about it. Let $C_{\sharp}^{\infty}(Y)$ designate the space of $C^{\infty}$ functions on $\mathbb{R}^{n}$ that are $Y$-periodic.

Définition 1 (Allaire [1]) Let $\left(u^{\varepsilon}\right)_{\varepsilon>0}$ be a family of $L^{2}(\omega)$ and $u \in L^{2}(\omega \times Y)$. We say that $\left(u^{\varepsilon}\right)_{\varepsilon>0}$ two-scale converges to $u$ if for any $v \in \mathcal{D}\left(\omega ; C_{\sharp}^{\infty}(Y)\right)$

$$
\lim _{\varepsilon \rightarrow 0} \int_{\omega} u^{\varepsilon}(\hat{x}) v(\hat{x}, \hat{x} / \varepsilon) \mathrm{d} \hat{x}=\int_{\omega \times Y} u(\hat{x}, y) v(\hat{x}, y) \mathrm{d} \hat{x} \mathrm{~d} y .
$$

The important fact here is that for any bounded family in $L^{2}(\omega)$, there is a subsequence that two-scale converges to some limit $u$. Two-scale convergence is a more accurate notion than usual weak convergence, in the sense that the two-scale converging family also weakly converges to $\int_{Y} u(., y) \mathrm{d} y$.

Since we need two-scale convergence for functions defined on $\omega_{1}^{\varepsilon}$, we also use the following practical definition. 
Définition 2 A family $\left(u^{\varepsilon}\right)_{\varepsilon>0}$ of $L^{2}\left(\omega_{1}^{\varepsilon}\right)$ is said to two-scale converge to a limit $u$ in $L^{2}\left(\omega \times Y_{1}\right)$ if $u \in L^{2}\left(\omega \times Y_{1}\right)$ and $\left(P^{\varepsilon} u^{\varepsilon}\right)$ two-scale converges to $P u$ in the sense of definition 1, where $P^{\varepsilon}$ and $P$ designate the extension by 0 of functions on $\omega_{1}^{\varepsilon}$ to functions on $\omega$ and of functions on $\omega \times Y_{1}$ to functions $\omega \times Y$ respectively.

In our problems, there are no oscillations in the $x_{3}$-direction. Still, the above definitions evidently apply with $x_{3}$ as a dummy variable (as it is the case for the time variables $t$ in other contexts). So in what follows when writing that $\left(u^{\varepsilon}\right)$ of $L^{2}(\Omega)$ two-scale converges to $u$ in $L^{2}(\Omega \times Y)$, we mean that for any $v \in \mathcal{D}\left(\Omega ; C_{\sharp}^{\infty}(Y)\right)$,

$$
\lim _{\varepsilon \rightarrow 0} \int_{\Omega} u^{\varepsilon}(x) v(x, \hat{x} / \varepsilon) \mathrm{d} x=\int_{\Omega \times Y} u(x, y) v(x, y) \mathrm{d} x \mathrm{~d} y,
$$

and similarly for $\left(u^{\varepsilon}\right)$ in $L^{2}\left(\Omega \times Y_{1}\right)$.

Remark that the convergences in Definitions 1 and 2 are weak convergences. In general, the two-scale limit of a product is not the product of the two-scale limits. An additional assumption is needed. Let $\left(u^{\varepsilon}\right)$ and $\left(v^{\varepsilon}\right)$ be two two-scale converging family in $L^{2}(\Omega)$ with limits $u$ and $v$ respectively. Then, if in addition

$$
\lim _{\varepsilon \rightarrow 0}\left\|u^{\varepsilon}\right\|_{L^{2}(\omega)}=\|u\|_{L^{2}(\omega \times Y)},
$$

the following statement hold true for any regular test functions $\varphi$ :

$$
\lim _{\varepsilon \rightarrow 0} \int_{\omega} u^{\varepsilon}(\hat{x}) v^{\varepsilon}(\hat{x}) \varphi(\hat{x}, \hat{x} / \varepsilon) \mathrm{d} \hat{x}=\int_{\omega \times Y} u(\hat{x}, y) \varphi(\hat{x}, y) \varphi \mathrm{d} \hat{x} \mathrm{~d} y .
$$

Assumption (15) is a kind of strong two-scale convergence notion. See for example Theorem 1.8 and the proof of Theorem 2.3 in Allaire [1] for a proof of (16).

4.1 Assumptions on the data and first convergence results. The tensors $\mathbf{R}^{\varepsilon}$, $\mathbf{d}^{\varepsilon}$ and $\mathbf{c}^{\varepsilon}$ constituting the stiffness-piezoelectricity-permitivity tensor $\mathcal{R}^{\varepsilon}$ are assumed to satisfy (5) and

$$
\left\{\begin{array}{l}
\left(\mathcal{R}^{\varepsilon}\right) \in \mathbf{L}^{\infty}(\Omega) \text { and two-scale converges to some } \mathcal{R} \in \mathbf{L}^{\infty}(\Omega \times Y), \\
\left\|\mathcal{R}^{\varepsilon}\right\|_{\mathbf{L}^{\infty}(\Omega)} \leq C, \mathcal{R}^{\varepsilon} \text { does not depend on } x_{3}, \\
\forall \mathbf{K}=\left(K_{i j}\right) \in \mathbb{R}^{3 \times 3} \text { with } K_{i j}=K_{j i}, \quad \mathbf{K} \mathbf{R}^{\varepsilon}{ }^{t} \mathbf{K} \geq c\|\mathbf{K}\|^{2} \text { a.e. in } \omega, \\
\forall \mathbf{L} \in \mathbb{R}^{3},{ }^{t} \mathbf{L} \mathbf{c}^{\varepsilon} \mathbf{L} \geq c\|\mathbf{L}\|^{2} \text { a.e. in } \omega_{1}^{\varepsilon},
\end{array}\right.
$$

and

$$
\lim _{\varepsilon \rightarrow 0}\left\|\mathcal{R}^{\varepsilon}\right\|_{\mathbf{L}^{2}(\omega)}=\|\mathcal{R}\|_{\mathbf{L}^{2}(\omega \times Y)} .
$$

Remark: As pointed above, assumption (18) is a rather vague, but general assumption that allows to go to the limit in product of two-scale converging functions; namely 
$\left(\mathbf{M}^{a}\left(\mathbf{U}^{b}\right)\right)$ and $\left(\mathcal{R}^{\varepsilon}\right)$. It is of course fulfilled by data satisfying classical periodicity conditions, for instance $\mathcal{R}^{\varepsilon}(\hat{x})=\mathcal{R}(\hat{x}, \hat{x} / \varepsilon)$ where $\mathcal{R}$ is a given tensor in $\mathbf{L}^{\infty}\left(\omega ; C_{\sharp}(Y)\right)$, or also, which is more relevant in the present work, in $\mathbf{C}^{\infty}\left(\omega ; L_{\sharp}^{\infty}(Y)\right)$. See Allaire [1] if necessary.

Coercivity for $\mathbf{c}^{\varepsilon}$ and $\mathbf{R}^{\varepsilon}$, together with the symmetry assumptions (5) for $\mathbf{d}$, implies coercivity for $\mathcal{R}^{\varepsilon}$. Conversely, two-scale convergence for $\mathcal{R}^{\varepsilon}$ implies two-scale convergence for $\mathbf{R}^{\varepsilon}, \mathbf{c}^{\varepsilon}$ and $\mathbf{d}^{\varepsilon}$. The corresponding limits are denoted by $\mathbf{R}, \mathbf{c}$ and $\mathbf{d}$. The other data are assumed to satisfy

$$
\left\{\begin{array}{l}
\mathbf{f}^{b} \in \mathbf{L}^{2}(\Omega), \mathbf{g}^{b} \in \mathbf{H}^{1 / 2}\left(\Gamma_{N}\right) \\
\left(\mathbf{f}^{b}\right) \text { converges weakly in } \mathbf{L}^{2}(\Omega) \text { to some limit } \mathbf{f} \\
\left(\mathbf{g}^{b}\right) \text { converges weakly in } \mathbf{L}^{2}\left(\Gamma_{N}\right) \text { to some limit } \mathbf{g}
\end{array}\right.
$$

$$
\left\{\begin{array}{l}
h^{b}, \varphi_{m}^{b} \text { and } \varphi_{c}^{b} \text { are constant on each inclusion, } \\
\left(h^{b}\right) \text { two scale-converges in } L^{2}\left(\omega \times Y_{1}\right) \text { to some limit } h \in L^{2}(\omega), \\
\left(\varphi_{m}^{b}\right) \text { two-scale converges in } L^{2}\left(\omega \times Y_{1}\right) \text { to some limit } \varphi_{m} \in H^{1}(\omega), \\
\left(\varphi_{c}^{b}\right) \text { two-scale converges in } L^{2}\left(\omega \times Y_{1}\right) \text { to some limit } \varphi_{c} \in L^{2}(\omega)
\end{array}\right.
$$

We observe that, because $\varphi_{c}^{b}, \varphi_{m}^{b}$ and $h^{b}$ are constant on each inclusion, their two-scale limits do not depend on $y$ in $Y_{1}$.

Last, let us introduce the space of Kirchhoff-Love's displacement fields:

$$
\begin{aligned}
\mathbf{V}_{K L} & =\left\{\mathbf{v} \in \mathbf{H}^{1}(\Omega) ; \mathbf{v}=\mathbf{0} \text { on } \Gamma_{D},\left(s_{i 3}(\mathbf{v})\right)_{i=1,2,3}=\mathbf{0}\right\} \\
& =\left\{\left(\bar{v}_{1}-x_{3} \partial_{1} v_{3}, \bar{v}_{2}-x_{3} \partial_{2} v_{3}, v_{3}\right) ; \quad \overline{\mathbf{v}}:=\left(\bar{v}_{1}, \bar{v}_{2}\right) \in \mathbf{H}^{1}(\omega), v_{3} \in H^{2}(\omega),\right. \\
& \left.\overline{\mathbf{v}}=\nabla_{\hat{x}} v_{3}=\mathbf{0} \text { and } v_{3}=0 \text { on } \Gamma_{D}\right\} .
\end{aligned}
$$

The following lemma was proved in [7], the mean-operator $\mathcal{M}$ being defined in (1).

Lemma 4.1. If assumptions $(5,17,19,20)$ and conventions $(6,10,11)$ hold then

(i) for each fixed $b$ there is a unique solution to (14);

(ii) $\left\|\mathbf{K}^{a}\left(\mathbf{u}^{b}\right)\right\|_{\mathbf{L}^{2}(\Omega)}+\left\|\mathbf{L}^{a}\left(\varphi^{b}\right)\right\|_{\mathbf{L}^{2}\left(\Omega_{1}^{\varepsilon}\right)}+\sqrt{G_{1}}\left\|\nabla_{\hat{x}}^{\varepsilon} \mathcal{M}\left(L_{3}^{a}\left(\varphi^{b}\right)\right)\right\|_{\mathbf{L}^{2}\left(\Omega_{1}^{\varepsilon}\right)} \leq C ;$

(iii) there exists $\mathbf{M}=(\mathbf{K}, \mathbf{L}) \in\left(L^{2}(\Omega \times Y)\right)^{7} \times\left(L^{2}\left(\Omega \times Y_{1}\right)\right)^{3}$ such that $\left(\mathbf{M}^{a}\left(\mathbf{U}^{b}\right)\right)$ two-scale converges to $\mathbf{M}$ in $\mathbf{L}^{2}(\Omega \times Y) \times \mathbf{L}^{2}\left(\Omega \times Y_{1}\right)$;

(iv) there exists $\mathbf{u} \in \mathbf{V}_{K L}$ and $\mathbf{u}^{1}=\left(u_{1}^{1}, u_{2}^{1}, 0\right)$ with $u_{1}^{1}, u_{2}^{1} \in L^{2}\left(\Omega ; H_{\sharp}^{1}(Y) / \mathbb{R}\right)$ such that

$\left(\mathbf{u}^{b}\right)$ converges weakly to $\mathbf{u}$ in $\mathbf{H}^{1}(\Omega)$,

$\left(\nabla_{\hat{x}} \mathbf{u}^{b}\right)$ two-scale converges to $\nabla_{\hat{x}} \mathbf{u}+\nabla_{y} \mathbf{u}^{1}$ in $\mathbf{L}^{2}\left(\Omega \times Y_{1}\right)$,

$\left(\partial_{3} \mathbf{u}^{b}\right)$ two-scale converges to $\partial_{3} \mathbf{u}$ in $\mathbf{L}^{2}\left(\Omega \times Y_{1}\right)$;

(v) $\left(\varphi^{b}\right)$ two-scale converges to $\varphi_{m}$ in $L^{2}\left(\Omega \times Y_{1}\right)$; 
(vi) there exists $\varphi^{1} \in L^{2}\left(\Omega ; H^{1}\left(Y_{1}\right) / \mathbb{R}\right)$ such that ${ }^{t}\left(L_{1}, L_{2}\right)=\nabla_{y} \varphi^{1}$,

(vii) $\mathcal{M}\left(L_{3}\right)$ is independent of $y$ and for Dirichlet conditions $\mathcal{M}\left(L_{3}\right)=\varphi_{c}$;

(viii) In the case of nonlocal mixed conditions, $\mathcal{M}\left(L_{3}\right) \in H^{1}(\omega)$ and $\left(\nabla_{\hat{x}}^{\varepsilon} \mathcal{M}\left(L_{3}^{a}\left(\varphi^{b}\right)\right)\right)$ two-scale converges to $\nabla_{\hat{x}} \mathcal{M}\left(L_{3}\right)$ in $\mathbf{L}^{2}\left(\Omega \times Y_{1}\right)$.

5. Limit Models I - Effective composite plate models when the thickness is small with respect to the size of the inclusions.

This section is devoted to the statement of the two-dimensional composite plate models when the thickness of the plate is much smaller than the size of the inclusions, that is when $\varepsilon, a$ and $a / \varepsilon$ tend together to zero. The models are derived by coupling the homogenization method and the asymptotic method for plates. Consequently, they present characteristics of both approaches. Since no other situation occurs when $a$ is small with respect to $\varepsilon$, it would be a posteriori equivalent to derive first the two-dimensional plate model and then to apply the method of homogenization to obtain a homogeneous two dimensional plate model.

The results are summarized in Theorem 5.1 below, which is given after introducing a few notations: the projections involved by the plate approach, and the local problems and homogenized tensors involved by the homogenization process. The proof is postponed to Section 7 .

\subsection{Notations related to plate theory}

The stiffness - piezoelectric - permittivity coefficients of the two dimensional plate model obtained by eliminating the transverse components are as follows.

$$
\left\{\begin{array}{l}
\Pi \text { and } \Pi_{1} \text { are respectively the projections from }\left(L^{2}(\Omega \times Y)\right)^{7} \times\left(L^{2}\left(\Omega \times Y_{1}\right)\right)^{3} \\
\text { on its subspaces }\left\{\left(\mathbf{0}_{4},\left(K_{i 3}\right)_{i=1 . .3}, \mathbf{0}_{2}, L_{3}\right)\right\} \text { and }\left\{\left(\mathbf{0}_{9}, L_{3}\right)\right\}, \Pi_{2}=\Pi-\Pi_{1}, \\
\mathbf{T}_{\mathcal{N}}=-(\Pi \mathcal{R} \Pi)^{-1} \Pi \mathcal{R}, \mathbf{T}_{\mathcal{M}}=-\left(\Pi_{2} \mathcal{R} \Pi_{2}\right)^{-1} \Pi_{2} \mathcal{R}, \\
\mathcal{R}_{\mathcal{N}}=\left(\mathrm{Id}+{ }^{t} \mathbf{T}_{\mathcal{N}}\right) \mathcal{R}\left(\mathrm{Id}+\mathbf{T}_{\mathcal{N}}\right), \mathcal{R}_{\mathcal{M}}=\left(\mathrm{Id}+{ }^{t} \mathbf{T}_{\mathcal{M}}\right)\left(\mathcal{R}+2 G \Pi_{1}\right)\left(\operatorname{Id}+\mathbf{T}_{\mathcal{M}}\right) .
\end{array}\right.
$$

Remark: with notations like $(\Pi \mathcal{R} \Pi)^{-1}$, we mean the inverse application of $\Pi \mathcal{R} \Pi$ as an application onto $\left\{\left(\mathbf{0}_{4},\left(K_{i 3}\right)_{i=1 . .3}, \mathbf{0}_{2}, L_{3}\right)\right\}$ (for the sake of simplicity in the notation, $\Pi, \Pi_{2}$ are identified with their transposed applications).

\subsection{Notations related to homogenization theory}

Let $R_{\mathcal{M} \alpha \beta \gamma \delta}$, and $R_{\mathcal{N} \alpha \beta \gamma \delta}$ denote the relevant coefficients of the tensors $\mathcal{R}_{\mathcal{M}}, \mathcal{R}_{\mathcal{N}}$ and $\mathcal{R}_{\mathcal{M}}^{\text {Mix }}$ written on the format (13). Let

$$
H_{\sharp}^{2}(Y)=\left\{v \in H^{2}(Y) ; v \text { and } \nabla v \text { are } Y-\text { periodic }\right\} .
$$

For any $\mathbf{v} \in \mathbf{H}^{1}(Y)$, let

$$
S_{\alpha \beta}(\mathbf{v})=\frac{1}{2}\left(\partial_{y_{\alpha}} v_{\beta}+\partial_{y_{\beta}} v_{\alpha}\right)
$$


Let $\left(\mathbf{u}_{\mathcal{M}}^{\gamma \delta}, u_{\mathcal{N} 3}^{\gamma \delta}\right) \in\left(H_{\sharp}^{1}(Y)\right)^{2} \times H_{\sharp}^{2}(Y)$, for $\gamma, \delta \in\{1,2\}$, be the solutions of

$$
\left\{\begin{array}{l}
\forall \mathbf{v} \in\left(H_{\sharp}^{1}(Y)\right)^{2}, \quad \int_{Y} S_{\alpha \beta}(\mathbf{v}) R_{\mathcal{M} \alpha \beta \lambda \mu} S_{\lambda \mu}\left(\mathbf{u}_{\mathcal{M}}^{\gamma \delta}\right) \mathrm{d} y=-\int_{Y} S_{\alpha \beta}(\mathbf{v}) R_{\mathcal{M} \alpha \beta \gamma \delta} \mathrm{d} y, \\
\forall v_{3} \in H_{\sharp}^{2}(Y), \quad \int_{Y} \partial_{y_{\alpha} y_{\beta}}^{2} v_{3} R_{\mathcal{N} \alpha \beta \lambda \mu} \partial_{y_{\lambda} y_{\mu}}^{2} u_{\mathcal{N} 3}^{\gamma \delta} \mathrm{d} y=-\int_{Y} \partial_{y_{\alpha} y_{\beta}}^{2} v_{3} R_{\mathcal{N} \alpha \beta \gamma \delta} \mathrm{d} y .
\end{array}\right.
$$

Let $\mathbf{u}_{\mathcal{M}}^{3} \in\left(H_{\sharp}^{1}(Y)\right)^{2}$ be the solution of

$$
\forall \mathbf{v} \in\left(H_{\sharp}^{1}(Y)\right)^{2}, \quad \int_{Y} S_{\alpha \beta}(\mathbf{v}) R_{\mathcal{M} \alpha \beta \lambda \mu} S_{\lambda \mu}\left(\mathbf{u}_{\mathcal{M}}^{3}\right) \mathrm{d} y=-\int_{Y} d_{\mathcal{M} 3 \alpha \beta} S_{\alpha \beta}(\mathbf{v}) \mathrm{d} y .
$$

The effective tensors $R_{\mathcal{M}}^{H}, R_{\mathcal{N}}^{H}, d_{\mathcal{M}}^{H}, e_{\mathcal{M}}^{H}$ and $c_{33}^{H}$ are then defined by

$$
\begin{aligned}
& R_{\mathcal{N} \gamma \delta \rho \xi}^{H}=\int_{Y}\left(\delta_{\alpha \beta, \gamma \delta}+\partial_{y_{\alpha} y_{\beta}}^{2} u_{\mathcal{N}}^{\gamma \delta}\right) R_{\mathcal{N} \alpha \beta \lambda \mu}\left(\delta_{\lambda \mu, \rho \xi}+\partial_{y_{\lambda} y_{\mu}}^{2} u_{\mathcal{N}}^{\rho \xi}\right) \mathrm{d} y \\
& \left\{\begin{array}{c}
\left(\begin{array}{cc}
R_{\mathcal{M} \gamma \delta \rho \xi}^{H} & d_{\mathcal{M} 3 \gamma \delta}^{H} \\
e_{\mathcal{M} 3 \rho \xi}^{H} & c_{\mathcal{M} 33}^{H}
\end{array}\right)=\int_{Y}\left(\begin{array}{cc}
\delta_{\alpha \beta, \gamma \delta}+S_{\alpha \beta}\left(\mathbf{u}_{\mathcal{M}}^{\gamma \delta}\right) & 0 \\
S_{\lambda \mu}\left(\mathbf{u}_{\mathcal{M}}^{3}\right) & 1
\end{array}\right) \\
\left(\begin{array}{cc}
R_{\mathcal{M} \alpha \beta \lambda \mu} & d_{\mathcal{M} 3 \alpha \beta} \\
-d_{\mathcal{M} 3 \lambda \mu} & c_{\mathcal{M} 33}
\end{array}\right)\left(\begin{array}{cc}
\delta_{\lambda \mu, \rho \xi}+S_{\lambda \mu}\left(\mathbf{u}_{\mathcal{M}}^{\rho \xi}\right) & S_{\lambda \mu}\left(\mathbf{u}_{\mathcal{M}}^{3}\right) \\
0 & 1
\end{array}\right) \mathrm{d} y
\end{array}\right.
\end{aligned}
$$

where the notations $\delta_{\lambda \mu, \rho \xi}$ are Kronecker symbols, that is

$$
\begin{aligned}
\delta_{\lambda \mu, \rho \xi} & =1 \text { if } \lambda=\rho \text { and } \mu=\xi \\
& =0 \text { if not. }
\end{aligned}
$$

For local mixed conditions we also need

$$
R_{\mathcal{M} \alpha \beta \gamma \delta}^{H, l o c}=R_{\mathcal{M} \alpha \beta \gamma \delta}^{H}-\left(c_{\mathcal{M} 33}^{H}+2\left|Y_{1}\right| G\right)^{-1} d_{\mathcal{M} 3 \alpha \beta}^{H} e_{\mathcal{M} 3 \gamma \delta}^{H} .
$$

Let then

$$
\begin{gathered}
\ell_{u}(\mathbf{v})=\int_{\Omega} \mathbf{f} . \mathbf{v} \mathrm{d} x+\int_{\Gamma_{N}} \mathbf{g . v ~} \mathrm{d} s \\
\ell(\mathbf{v})=\left\{\begin{array}{l}
\ell_{u}(\mathbf{v})-2 \int_{\omega} s_{\alpha \beta}(\overline{\mathbf{v}}) d_{\mathcal{M} 3 \alpha \beta}^{H} \varphi_{c} \mathrm{~d} \hat{x} \quad \text { (Dirichlet cond.) } \\
\ell_{u}(\mathbf{v})-2 \int_{\omega} s_{\alpha \beta}(\overline{\mathbf{v}}) d_{\mathcal{M} 3 \alpha \beta}^{H}\left(c_{\mathcal{M} 33}^{H}+2\left|Y_{1}\right| G\right)^{-1}\left|Y_{1}\right| h \mathrm{~d} \hat{x} \quad \text { (local mixed cond.) } \\
\ell_{u}(\mathbf{v})+2\left|Y_{1}\right| \int_{\omega} \tilde{L}_{3} h \mathrm{~d} \hat{x} \text { (nonlocal mixed cond.). }
\end{array}\right.
\end{gathered}
$$

\subsection{Theorem 5.1: effective models}

The set $\mathbf{V}_{K L}$ of Kirchoff-Love displacement fields have been defined in (21). 
Theorem 5.1. Assume that the assumptions of Section 2.4 hold and that $a$, $\varepsilon$ and $a / \varepsilon$ tend together to zero,

(i) in the case of Dirichlet conditions $\left(\mathbf{u}^{b}\right)$ converges to

$$
\mathbf{u}=\left(\bar{u}_{1}-x_{3} \partial_{1} u_{3}, \bar{u}_{2}-x_{3} \partial_{2} u_{3}, u_{3}\right) \in \mathbf{V}_{K L}
$$

which is the unique solution in $\mathbf{V}_{K L}$ of

$$
\forall \mathbf{v} \in \mathbf{V}_{K L}, \quad \int_{\omega}\left(2 s_{\alpha \beta}(\overline{\mathbf{v}}) R_{\mathcal{M} \alpha \beta \gamma \delta}^{H} s_{\gamma \delta}(\overline{\mathbf{u}})+\frac{2}{3} \partial_{\alpha \beta}^{2} v_{3} R_{\mathcal{N} \alpha \beta \gamma \delta}^{H} \partial_{\gamma \delta}^{2} u_{3}\right) \mathrm{d} \hat{x}=\ell(\mathbf{v}) ;
$$

(ii) in the case of local mixed conditions, $\left(\mathbf{u}^{b}\right)$ converges to $\mathbf{u}$ which is the unique solution in $V_{K L}$ of

$$
\forall \mathbf{v} \in \mathbf{V}_{K L}, \quad \int_{\omega}\left(2 s_{\alpha \beta}(\overline{\mathbf{v}}) R_{\mathcal{M} \alpha \beta \gamma \delta}^{H, l o c} s_{\gamma \delta}(\overline{\mathbf{u}})+\frac{2}{3} \partial_{\alpha \beta}^{2} v_{3} R_{\mathcal{N} \alpha \beta \gamma \delta}^{H} \partial_{\gamma \delta}^{2} u_{3}\right) \mathrm{d} \hat{x}=\ell(\mathbf{v}) ;
$$

(iii) for nonlocal mixed conditions, $\left(\mathbf{u}^{b}, \mathcal{M}\left(L_{3}^{a}\left(\varphi^{b}\right)\right)\right)$ converges to

$$
\left(\mathbf{u}, L_{3}^{0}\right)=\left(\bar{u}_{1}-x_{3} \partial_{1} u_{3}, \bar{u}_{2}-x_{3} \partial_{2} u_{3}, u_{3}, L_{3}^{0}\right) \in \mathbf{V}_{K L} \times H^{1}(\omega)
$$

which is the unique solution of

$$
\left\{\begin{array}{c}
\forall\left(\mathbf{v}, \tilde{L}_{3}\right)=\left(\bar{v}_{1}-x_{3} \partial_{1} v_{3}, \bar{v}_{2}-x_{3} \partial_{2} v_{3}, v_{3}, \tilde{L}_{3}\right) \in \mathbf{V}_{K L} \times H^{1}(\omega) \\
\int_{\omega}\left(2\left(s_{\alpha \beta}(\overline{\mathbf{v}}), \tilde{L}_{3}\right)\left(\begin{array}{cc}
R_{\mathcal{M} \alpha \beta \gamma \delta}^{H} & d_{\mathcal{M} 3 \alpha \beta}^{H} \\
e_{\mathcal{M} 3 \gamma \delta}^{H} & c_{\mathcal{M} 33}^{H}+2\left|Y_{1}\right| G
\end{array}\right)\left(\begin{array}{c}
s_{\gamma \delta}(\overline{\mathbf{u}}) \\
L_{3}^{0}
\end{array}\right)\right) \mathrm{d} \hat{x} \\
+\int_{\omega}\left(4\left|Y_{1}\right| G_{1} \partial_{\alpha} \tilde{L}_{3} \partial_{\alpha} L_{3}^{0}+\frac{2}{3} \partial_{\alpha \beta}^{2} v_{3} R_{\mathcal{N} \alpha \beta \gamma \delta}^{H} \partial_{\gamma \delta}^{2} u_{3}\right) \mathrm{d} \hat{x}=\ell(\mathbf{v}) .
\end{array}\right.
$$

Remarks: (i) Since elasticity coefficients are constant in the thickness, the membrane and the flexion models are discoupled as for a single-layer elastic plate.

(ii) for Dirichlet, and local mixed conditions, the limit model has the same form as the elastic plate model, the influence of piezoelectric inclusion only appears in the definition of the effective coefficients and as a source term.

(iii) The piezoelectric force operates only in the membrane model as for the single-layer piezoelectric plate. A piezoelectric force may be generated in the flexion model by a torque in a multi-layer piezoelectric plate.

(iv) In a thin plate with an imposed voltage $\varphi_{c}$ on the upper face of a piezoelectric inclusion $\omega_{P}$, the resulting force is concentrated along the boundary of the inclusion and may be expressed as a distribution $v \mapsto \sum_{\alpha, \beta=1}^{2}\left\langle d_{\alpha \beta} \varphi_{c}, s_{\alpha \beta}(v)\right\rangle_{\omega_{P}}$ where $v$ is a regular test function, $d$ the piezoelectric coefficient, and $\langle., .\rangle_{\omega_{P}}$ the distribution bracket over the mean surface of the inclusion. For a periodic distribution of piezoelectric inclusions with an imposed voltage $\varphi_{c}$ on the upper faces, as considered in this paper, the resulting 
force is distributed over the complete mean surface $\omega$ and is still a distribution $v \mapsto$ $\sum_{\alpha, \beta=1}^{2}\left\langle d_{\alpha \beta} \varphi_{c}, s_{\alpha \beta}(v)\right\rangle_{\omega}$ because in general, the distributed voltage $\varphi_{c} \in L^{2}(\omega)$ has no regularity.

(v) For nonlocal conditions, the presence of derivatives in the term $\int_{\omega} \partial_{\alpha} \tilde{L}_{3} \partial_{\alpha} L_{3} \mathrm{~d} \hat{x}$ is due to the electric circuits connecting neighbouring cells, which results in a regularization of the transverse component of the electric field $L_{3}$ and in a direct nontrivial coupling between mechanical and electrical effects. Note that one may a priori choose the form of the differential operator on $L_{3}$ by choosing the way to connect the upper faces of the inclusions to each others. This last point is interresting in view of controlling the structure.

(vi) For the case of local mixed conditions, the local circuit, i.e. without connections between cells, allows elimination of $L_{3}$ from the effective equations. Even if it is actuated by the current source $h$, the piezoelectric force is similar to the case of an imposed voltage with $\varphi_{c}=\left|Y_{1}\right| h /\left(c_{\mathcal{M} 33}^{H}+2\left|Y_{1}\right| G\right)$ where $1 /\left(c_{\mathcal{M} 33}^{H}+2\left|Y_{1}\right| G\right)$ is the impedance of the local circuit.

(vii) It would also be possible to keep the voltage $L_{3}$ in the model with local mixed conditions. The resulting model would be similar to the model with nonlocal condition, i.e. with the current source $h$, but with $G_{1}=0$. This formulation is most suited for extension to dynamic problems where the admittance $G$ is generally an integro-differential operator in time.

\section{Limit Models II - Effective composite plate models when $a \sim \varepsilon$.}

This section is devoted to the statement of the two-dimensional limit models when $a$ and $\varepsilon$ tend to zero while $a / \varepsilon$ tends to a finite positive limit. Then, there is no loss of generality in assuming that $a / \varepsilon$ tends to 1 . If $a / \varepsilon$ tends to any other positive number $\ell \in \mathbb{R}^{+*}$, one just has to normalize the coordinates $\left(x_{1}, x_{2}\right)$ in an appropriate way. However $\ell$ must be not too far from 1. If not, one of the two other models, $a / \varepsilon \rightarrow 0$ if $\ell<<1, \varepsilon / a \rightarrow 0$ if $\ell>>1$, would be to consider.

The results are summarized in Theorem 6.1 below, which is given after introducing necessary preliminary notations, in particular the local problems to be solved to compute the effective coefficients of the limit models. The proof is postponed to Section 8.

6.1 Notations related to the microstructure. Because $a$ and $\varepsilon$ are of the same order of magnitude, we have here a unique three-dimensional microstructure containing both the $y$ and $x_{3}$ directions. Let us introduce appropriate notations

$$
\begin{gathered}
Z=Y \times]-1,1\left[, Z_{1}=Y_{1} \times\right]-1,1\left[, z=\left(y_{1}, y_{2}, x_{3}\right),\right. \\
\Gamma^{+}=Y_{1} \times\{1\}, \Gamma^{-}=Y_{1} \times\{-1\}, \\
\mathbf{W}^{1}=\left(L^{2}(]-1,1\left[; H_{\sharp}^{1}(Y)\right) \cap H^{1}(Z)\right)^{3} \times \Psi^{1} \text { where } \\
\Psi^{1}=\left\{\psi \in H^{1}\left(Z_{1}\right) ; \psi=0 \text { on } \Gamma^{-} \cup \Gamma^{+}\right\} .
\end{gathered}
$$




$$
\begin{aligned}
& \left.\forall \mathbf{V}^{1}=\left(\mathbf{v}^{1}, \psi^{1}\right) \in \mathbf{W}^{1}, \mathbf{M}^{1}(\mathbf{V})=\left(\left(S_{i j}\left(\mathbf{v}^{1}\right)\right)_{i, j=1 . .3}, \nabla_{z} \psi^{1}\right)\right) \\
& \text { where } S_{i j}\left(\mathbf{v}^{1}\right)=\frac{1}{2}\left(\partial_{z_{i}} v_{j}^{1}+\partial_{z_{j}} v_{i}^{1}\right) .
\end{aligned}
$$

Let $\mathbf{U}_{\mathcal{M}}^{\lambda \mu}=\left(\mathbf{u}_{\mathcal{M}}^{\lambda \mu}, \varphi_{\mathcal{M}}^{\lambda \mu}\right) \in \mathbf{W}^{1}$ designates for any $(\lambda, \mu) \in\{1,2\}^{2}$ the solutions to

$$
\forall \mathbf{V}^{1} \in \mathbf{W}^{1}, \int_{Z} \mathbf{M}^{1}\left(\mathbf{V}^{1}\right) \mathcal{R}{ }^{t} \mathbf{M}^{1}\left(\mathbf{U}_{\mathcal{M}}^{\lambda \mu}\right) \mathrm{d} z=-\int_{Z} \mathbf{M}^{1}\left(\mathbf{V}^{1}\right){ }^{t} \mathbf{X}_{\lambda \mu} \mathrm{d} z
$$

where $\mathbf{X}_{\lambda \mu}=\left(R_{\alpha \beta \lambda \mu}, 2 R_{\alpha 3 \lambda \mu}, R_{33 \lambda \mu},-d_{\alpha \lambda \mu},-d_{3 \lambda \mu}\right)$ is for the first column of $\mathcal{R}$. Similarly, let $\mathbf{U}_{\mathcal{N}}^{\lambda \mu}=\left(\mathbf{u}_{\mathcal{N}}^{\lambda \mu}, \varphi_{\mathcal{N}}^{\lambda \mu}\right) \in \mathbf{W}^{1}$ be solutions to

$$
\forall \mathbf{V}^{1} \in \mathbf{W}^{1}, \int_{Z} \mathbf{M}^{1}\left(\mathbf{V}^{1}\right) \mathcal{R}{ }^{t} \mathbf{M}^{1}\left(\mathbf{U}_{\mathcal{N}}^{\lambda \mu}\right) \mathrm{d} z=\int_{Z} x_{3} \mathbf{M}^{1}\left(\mathbf{V}^{1}\right){ }^{t} \mathbf{X}_{\lambda \mu} \mathrm{d} z,
$$

and $\mathbf{U}^{3}=\left(\mathbf{u}^{3}, \varphi^{3}\right) \in \mathbf{W}^{1}$ be solution to

$$
\forall \mathbf{V}^{1} \in \mathbf{W}^{1}, \int_{Z} \mathbf{M}^{1}\left(\mathbf{V}^{1}\right) \mathcal{R}{ }^{t} \mathbf{M}^{1}\left(\mathbf{U}^{3}\right) \mathrm{d} z=\int_{Z} \mathbf{M}^{1}\left(\mathbf{V}^{1}\right){ }^{t} \mathbf{X}_{3} \mathrm{~d} z
$$

where $\mathbf{X}_{3}=\left(d_{3 \alpha \beta}, 2 d_{3 \alpha 3}, d_{333}, c_{\alpha 3}, c_{33}\right)$ stands for the last column of $\mathcal{R}$.

The effective coefficients are then given by:

$$
\begin{aligned}
& \left\{\begin{array}{l}
R_{\mathcal{M M} \lambda \mu \rho \xi}^{H}=\int_{Z}\left(\mathbf{E}^{\lambda \mu}+\mathbf{M}^{1}\left(\mathbf{U}_{\mathcal{M}}^{\lambda \mu}\right)\right) \mathcal{R}^{t}\left(\mathbf{E}^{\rho \xi}+\mathbf{M}^{1}\left(\mathbf{U}_{\mathcal{M}}^{\rho \xi}\right)\right) \mathrm{d} z \\
R_{\mathcal{M N} \lambda \mu \rho \xi}^{H}=\int_{Z}\left(\mathbf{E}^{\lambda \mu}+\mathbf{M}^{1}\left(\mathbf{U}_{\mathcal{M}}^{\lambda \mu}\right)\right) \mathcal{R}^{t}\left(-x_{3} \mathbf{E}^{\rho \xi}+\mathbf{M}^{1}\left(\mathbf{U}_{\mathcal{N}}^{\rho \xi}\right)\right) \mathrm{d} z \\
R_{\mathcal{N} \mathcal{M} \lambda \mu \rho \xi}^{H}=\int_{Z}\left(-x_{3} \mathbf{E}^{\lambda \mu}+\mathbf{M}^{1}\left(\mathbf{U}_{\mathcal{N}}^{\lambda \mu}\right)\right) \mathcal{R}^{t}\left(\mathbf{E}^{\rho \xi}+\mathbf{M}^{1}\left(\mathbf{U}_{\mathcal{M}}^{\rho \xi}\right)\right) \mathrm{d} z \\
R_{\mathcal{N} \mathcal{N} \lambda \mu \rho \xi}^{H}=\int_{Z}\left(-x_{3} \mathbf{E}^{\lambda \mu}+\mathbf{M}^{1}\left(\mathbf{U}_{\mathcal{N}}^{\lambda \mu}\right)\right) \mathcal{R}^{t}\left(-x_{3} \mathbf{E}^{\rho \xi}+\mathbf{M}^{1}\left(\mathbf{U}_{\mathcal{N}}^{\rho \xi}\right)\right) \mathrm{d} z \\
d_{\mathcal{N} \mathcal{M} 3 \lambda \mu}^{H}=\int_{Z}\left(-x_{3} \mathbf{E}^{\lambda \mu}+\mathbf{M}^{1}\left(\mathbf{U}_{\mathcal{N}}^{\lambda \mu}\right)\right) \mathcal{R}^{t}\left(\mathbf{b}+\mathbf{M}^{1}\left(\mathbf{U}^{3}\right)\right) \mathrm{d} z \\
e_{\mathcal{M} \mathcal{M} 3 \alpha \beta}^{H}=\int_{Z}\left(\mathbf{b}+\mathbf{M}^{1}\left(\mathbf{U}^{3}\right)\right) \mathcal{R}^{t}\left(\mathbf{E}^{\alpha \beta}+\mathbf{M}^{1}\left(\mathbf{U}_{\mathcal{M}}^{\alpha \beta}\right)\right) \mathrm{d} z \\
e_{\mathcal{M} \mathcal{N} 3 \alpha \beta}^{H}=\int_{Z}\left(\mathbf{b}+\mathbf{M}^{1}\left(\mathbf{U}^{3}\right)\right) \mathcal{R}^{t}\left(-x_{3} \mathbf{E}^{\alpha \beta}+\mathbf{M}^{1}\left(\mathbf{U}_{\mathcal{N}}^{\alpha \beta}\right)\right) \mathrm{d} z \\
c_{\mathcal{M} \mathcal{M} 33}^{H}=\int_{Z}\left(\mathbf{b}+\mathbf{M}^{1}\left(\mathbf{U}^{3}\right)\right) \mathcal{R}^{t}\left(\mathbf{b}+\mathbf{M}^{1}\left(\mathbf{U}^{3}\right)\right) \mathrm{d} z
\end{array}\right.
\end{aligned}
$$


where $\mathbf{E}^{\lambda \mu}=\left(\left(\delta_{\alpha \beta, \lambda \mu}\right)_{\alpha, \beta=1,2}, \mathbf{0}_{6}\right)$ and $\mathbf{b}=\left(\mathbf{0}_{9}, 1\right)$.

\subsection{Effective models: Theorem 6.1}

The set $\mathbf{V}_{K L}$ of Kirchoff-Love displacement fields have been defined in (21).

Theorem 6.1. Assume that the assumptions of Section 2.4 hold and in addition that $a, \varepsilon$ tend to zero and $a / \varepsilon$ tends to 1 then

(i) in the case of Dirichlet conditions, the limit $\mathbf{u}$ of $\left(\mathbf{u}^{b}\right)_{b>0}$ satisfies: $\mathbf{u}=\left(\bar{u}_{1}-x_{3} \partial_{1} u_{3}\right.$ $\left., \bar{u}_{2}-x_{3} \partial_{2} u_{3}, u_{3}\right) \in \mathbf{V}_{K L}$ and is the unique solution in $\mathbf{V}_{K L}$ of

$$
\left\{\begin{array}{r}
\forall \mathbf{v} \in V_{K L}, \quad \int_{\omega}\left(s_{\alpha \beta}(\overline{\mathbf{v}}), \partial_{\alpha \beta}^{2} v_{3}\right)\left(\begin{array}{cc}
R_{\mathcal{M M}}^{H} \alpha \beta \gamma \delta & R_{\mathcal{M N} \alpha \beta \gamma \delta}^{H} \\
R_{\mathcal{N} \mathcal{M} \alpha \beta \gamma \delta}^{H} & R_{\mathcal{N} \mathcal{N} \alpha \beta \gamma \delta}^{H}
\end{array}\right)\left(\begin{array}{c}
s_{\gamma \delta}(\overline{\mathbf{u}}) \\
\partial_{\gamma \delta}^{2} u_{3}
\end{array}\right) \mathrm{d} \hat{x}= \\
\ell_{u}(\mathbf{v})-\int_{\omega}\left(s_{\alpha \beta}(\overline{\mathbf{v}}), \partial_{\alpha \beta}^{2} v_{3}\right) d_{\mathcal{M} \mathcal{M} 3 \beta}^{H} \varphi_{c} \mathrm{~d} \hat{x}
\end{array}\right.
$$

(ii) in the case of mixed conditions, the limit $\mathbf{u}=\left(\bar{u}_{1}-x_{3} \partial_{1} u_{3}, \bar{u}_{2}-x_{3} \partial_{2} u_{3}, u_{3}\right)$ of $\left(\mathbf{u}^{b}\right)_{b>0}$ belongs to $\mathbf{V}_{K L}$, the limit $L_{3}^{0}$ of $\mathcal{M}\left(L_{3}\left(\varphi^{b}\right)\right)$ belongs to $L^{2}(\omega)$ or to $H^{1}(\omega)$ for nonlocal conditions, and $\left(\mathbf{u}, L_{3}^{0}\right)$ is the unique solution in $\mathbf{V}_{K L} \times L^{2}(\omega)$ or in $\mathbf{V}_{K L} \times H^{1}(\omega)$ for nonlocal conditions of

$$
\left\{\begin{aligned}
& \forall\left(\mathbf{v}, \widetilde{L}_{3}\right) \in \mathbf{V}_{K L} \times L^{2}(\omega)\left(\text { resp. } \mathbf{V}_{K L} \times H^{1}(\omega)\right) \\
& \int_{\omega}\left(s_{\alpha \beta}(\overline{\mathbf{v}}), \widetilde{L}_{3}, \partial_{\alpha \beta}^{2} v_{3}\right)\left(\begin{array}{ccc}
R_{\mathcal{M} \mathcal{M} \alpha \beta \gamma}^{H} & d_{\mathcal{M} \mathcal{M} 3 \alpha \beta}^{H} & R_{\mathcal{M} \mathcal{N} \alpha \beta \gamma \delta}^{H} \\
e_{\mathcal{M} \mathcal{M} 3 \alpha \beta}^{H} & c_{\mathcal{M} \mathcal{M} 33}^{H} & d_{\mathcal{M} \mathcal{N} 3 \alpha \beta}^{H} \\
R_{\mathcal{N} \mathcal{M} \alpha \beta \gamma \delta}^{H} & e_{\mathcal{M N} 3 \alpha \beta}^{H} & R_{\mathcal{N} \mathcal{N} \alpha \beta \gamma \delta}^{H}
\end{array}\right)\left(\begin{array}{c}
s_{\gamma \delta}(\overline{\mathbf{u}}) \\
L_{3}^{0} \\
\partial_{\gamma \delta}^{2} u_{3}
\end{array}\right) \mathrm{d} \hat{x} \\
& \quad+\int_{\omega}\left(4\left|Y_{1}\right|\left(G \widetilde{L}_{3} L_{3}^{0}+G_{1} \partial_{\alpha} \widetilde{L}_{3} \partial_{\alpha} L_{3}^{0}\right)\right) \mathrm{d} \hat{x}=\ell_{u}(\mathbf{v})+2\left|Y_{1}\right| \int_{\omega} \widetilde{L}_{3} h \mathrm{~d} \hat{x}
\end{aligned}\right.
$$

Remarks: (i) On the contrary to the model in Section 5, the membrane and flexion models are coupled. This comes from the presence of a microstructure which size is comparable to the plate thickness, and generates a complex displacement field at the scale of the plate thickness.

(ii) The other remarks (iii-vii) also hold for this model.

\section{Proof of Theorem 5.1.}

This section is devoted to the derivation of Theorem 5.1. The proof is based on the general results of Lemma 4.1. The proof consists in three steps. The first one is the characterization of the limit $\mathbf{M}$ of $\left(\mathbf{M}^{a}(\mathbf{V})\right)$ and is the aim of Section 7.1. The second one consists in the elimination of the $x_{3}$-variable as usual in plate theory. The last one is the elimination of the local variable as in homogenization theory and obtention of the effective models. The last two steps slightly differ from one boundary condition to the other, and thus are presented separately. In particular, in the case of local mixed conditions, the 
complete elimination of the transverse component is achieved after the elimination of the local variable. This two steps are presented in Section 7.2. The assumption that $\mathcal{R}$ does not depend on $x_{3}$ is widely used there to obtain simplifications and compute our limit models.

\subsection{Step 1: characterization of the limit M.}

In the forthcoming Lemma 7.4, the limit $\mathbf{M}$ is related to the limits $\mathbf{u}, \mathbf{u}^{1}$ and $\varphi^{1}$ defined in Lemma 4.1. The limit of equation (14) is given in Lemma 7.5. Suitable choices of tests functions $\mathbf{V}$ lead here to a list of equations corresponding to the various asymptotic levels of $\mathbf{M}^{a}(\mathbf{V})$. But first, we give à few additionnal notations, and technical results, in section 7.1.1.

\subsubsection{Further notations, and preliminary lemmas.}

Recall that $C_{\sharp}^{\infty}(Y)$ denotes the subspace of all $Y$-periodic functions of $C^{\infty}\left(\mathbb{R}^{2}\right)$. Similarly, let $C_{\sharp}^{\infty}\left(Y_{1}\right)$ denote the subspace of all $Y$-periodic functions of $C^{\infty}\left(\mathbb{Z}^{2}+Y_{1}\right)$. For functions $v$ on $\bar{\Omega} \times \mathbb{R}^{3}$ which are $Y$-periodic with respect to the second variable, we designate by $v^{\varepsilon}$ the function $x \longmapsto v(x, \hat{x} / \varepsilon)$. We also use test functions in

$$
\begin{gathered}
\mathbf{W}_{a d}^{1}=\left\{\left(\mathbf{v}^{1}, \psi^{1}\right) \in \mathbf{D}\left(\bar{\Omega}, C_{\sharp}^{\infty}(Y)\right) \times \Psi_{a d}^{1} ; \mathbf{v}^{1}=\mathbf{0} \text { on } \Gamma_{D} \times Y\right\}, \\
\mathbf{W}_{a d}=\left\{(\mathbf{v}, \psi) \in \mathbf{H}^{1}(\Omega) \times \Psi_{a d} ; \mathbf{v}=\mathbf{0} \text { on } \Gamma_{D}\right\},
\end{gathered}
$$

where

$$
\begin{aligned}
& \Psi_{a d}^{1}= \mathcal{D}(\omega \times]-1,1\left[; C_{\sharp}^{\infty}\left(Y_{1}\right)\right) \quad \text { for Dirichlet conditions, } \\
&\left.\left.=\left\{\psi^{1} \in \mathcal{D}(\omega \times]-1,1\right] ; C_{\sharp}^{\infty}\left(Y_{1}\right)\right) ; \psi^{1} \text { is constant for } x_{3}=1\right\} \quad \text { otherwise, } \\
& \Psi_{a d}=\mathcal{D}(\omega \times]-1,1[) \quad \text { for Dirichlet conditions, } \\
&\left.\quad=\{\psi \in \mathcal{D}(\omega \times]-1,1]) ; \psi \text { is constant for } x_{3}=1\right\} \quad \text { otherwise. }
\end{aligned}
$$

Functions in $\mathbf{W}_{a d}$ and in $\mathbf{W}_{a d}^{1}$ are admissible test functions for (14).

For $\mathbf{V}=(\mathbf{v}, \psi) \in \mathbf{W}_{a d}^{1}$, we have the natural decomposition of $\mathbf{M}^{a}\left(\mathbf{V}^{\varepsilon}\right)$ :

$$
\mathbf{M}^{a}\left(\mathbf{V}^{\varepsilon}\right)=\left(\mathbf{M}^{00}(\mathbf{V})\right)^{\varepsilon}+\frac{1}{\varepsilon}\left(\mathbf{M}^{10}(\mathbf{V})\right)^{\varepsilon}+\frac{1}{a}\left(\mathbf{M}^{01}(\mathbf{V})\right)^{\varepsilon}+\frac{1}{\varepsilon a}\left(\mathbf{M}^{11}(\mathbf{V})\right)^{\varepsilon}+\frac{1}{a^{2}}\left(\mathbf{M}^{02}(\mathbf{V})\right)^{\varepsilon}
$$

where

$$
\left\{\begin{array}{l}
\mathbf{M}^{00}(\mathbf{V})=\left(\left(s_{\alpha \beta}(\mathbf{v})\right)_{\alpha, \beta=1,2}, \mathbf{0}_{3},\left(\partial_{\alpha} \psi\right)_{\alpha=1,2}, 0\right) \\
\mathbf{M}^{10}(\mathbf{V})=\left(\left(S_{\alpha \beta}(\mathbf{v})\right)_{\alpha, \beta=1,2}, \mathbf{0}_{3},\left(\partial_{y_{\alpha}} \psi\right)_{\alpha=1,2}, 0\right) \\
\mathbf{M}^{01}(\mathbf{V})=\left(\mathbf{0}_{2 \times 2},\left(s_{\alpha 3}(\mathbf{v})\right)_{\alpha=1,2}, \mathbf{0}_{3}, \partial_{3} \psi\right) \\
\mathbf{M}^{11}(\mathbf{V})=\left(\mathbf{0}_{2 \times 2},\left(\frac{1}{2} \partial_{y_{\alpha}} v_{3}\right)_{\alpha=1,2}, \mathbf{0}_{4}\right) \\
\mathbf{M}^{02}(\mathbf{V})=\left(\mathbf{0}_{2 \times 2},, \mathbf{0}_{2}, s_{33}(\mathbf{v}), \mathbf{0}_{3}\right)
\end{array}\right.
$$


Notations $s_{i j}(\mathbf{v})$ and $S_{\alpha \beta}(\mathbf{v})$ have been defined in (4) and (23). Relevant subspaces $\mathbb{M}$, $\mathbb{M}^{0}, \mathbb{M}^{-1}$ and $\mathbb{M}^{-2}$ associated to this decomposition are defined by

$$
\left\{\begin{array}{l}
\mathbb{M}^{0}=\left\{\left(K_{\alpha \beta}, \mathbf{0}_{6}\right) ; K_{\alpha \beta}=s_{\alpha \beta}(\mathbf{v})+S_{\alpha \beta}\left(\mathbf{v}^{1}\right), \mathbf{v} \in \mathbf{V}_{K L}, \mathbf{v}^{1} \in \mathbf{V}_{K L}^{1}\right\}, \\
\mathbb{M}^{-1}=\left\{\left(\mathbf{0}_{2 \times 2}, K_{\alpha 3}, \mathbf{0}_{3}, L_{3}\right) ; K_{\alpha 3} \in L^{2}(\Omega \times Y), L_{3} \in L^{2}\left(\Omega \times Y_{1}\right),\right. \\
\left.\mathcal{M}\left(L_{3}\right) \text { is independant of } y ; \text { and } \mathcal{M}\left(L_{3}\right)=0 \text { for Dirichlet conditions }\right\} \\
\mathbb{M}^{-2}=\left\{\left(\mathbf{0}_{2 \times 2}, \mathbf{0}_{2}, K_{33}, \mathbf{0}_{3}\right) ; K_{33} \in L^{2}(\Omega \times Y)\right\} \\
\mathbb{M}=\mathbb{M}^{-2} \oplus \mathbb{M}^{-1} \oplus \mathbb{M}^{0} .
\end{array}\right.
$$

where

$$
\mathbf{V}_{K L}^{1}=\left\{\left(\left(\bar{v}_{\alpha}^{1}-x_{3} \partial_{y_{\alpha}} v_{3}^{2}\right)_{\alpha=1,2}, 0\right) ; \bar{v}_{\alpha}^{1} \in L^{2}\left(\omega ; H_{\sharp}^{1}(Y)\right), v_{3}^{2} \in L^{2}\left(\omega ; H_{\sharp}^{2}(Y)\right)\right\} .
$$

Our first lemma contains density results that will lead to a suitable weak formulation for the problem solved by $\mathbf{M}$.

Lemma 7.1. (i) The set $\left\{\mathbf{M}^{02}(\mathbf{V}) ; \mathbf{V} \in \mathbf{W}_{a d}^{1}\right\}$ is dense in $\mathbb{M}^{-2}$,

(ii) The set $\left\{\mathbf{M}^{01}(\mathbf{V}) ; \mathbf{V} \in \mathbf{W}_{a d}, v_{3}=0\right\}$ is dense in $\mathbb{M}^{-1}$,

(iii) The set $\left\{\mathbf{M}^{0}(\mathbf{V})+\mathbf{M}^{10}\left(\mathbf{V}^{1}\right) ;\left(\mathbf{V}, \mathbf{V}^{1}\right)=\left((\mathbf{v}, 0),\left(\mathbf{v}^{1}, 0\right)\right)\right.$ where $\left(\mathbf{V}, \mathbf{V}^{1}\right) \in \mathbf{W}_{a d}^{1},\left(\mathbf{v}, \mathbf{v}^{1}\right)$ $\left.\in \mathbf{V}_{K L} \times \mathbf{V}_{K L}^{1}\right\}$ is dense in $\mathbb{M}^{0}$.

Proof : (i) Let $K_{33} \in \mathcal{C}^{\infty}(\Omega \times Y)$ with compact support. This function $K_{33}$ can also be considered as a function of $\mathcal{D}\left(\bar{\Omega}, \mathcal{C}_{\sharp}^{\infty}(Y)\right)$ by letting

$$
\forall x \in \Omega, \forall y \in \mathbb{R}^{2}, K_{33}(x, y)=K_{33}\left(x, y^{\prime}\right),
$$

where $y^{\prime}$ is défined by: $y^{\prime} \in Y$ and $y-y^{\prime} \in \mathbb{Z}^{2}$. Then, $v_{3}$ defined by:

$$
v_{3}(x, y)=\int_{0}^{x_{3}} K_{33}(\hat{x}, t, y) \mathrm{d} t
$$

satisfies: $\left(\mathbf{0}_{2}, v_{3}, 0\right) \in \mathbf{W}_{a d}^{1}$ and $\partial_{3} v_{3}=K_{33}$. As the set of functions of $\mathcal{C}^{\infty}(\Omega \times Y)$ with compact support is dense in $L^{2}(\Omega \times Y)$, this proves that $\left\{\mathbf{M}^{02}(\mathbf{V}) ; \mathbf{V} \in \mathbf{W}_{a d}^{1}\right\}$ is dense in $\mathbb{M}^{-2}$.

(ii) The proof is similar for the first two terms $K_{\alpha 3}$ of the elements $\mathbb{M}^{-1}$. For the third term $L_{3}$ : let $L_{3}$ be a given function in $\mathcal{C}^{\infty}\left(\Omega \times Y_{1}\right)$ with compact support; we also assume that $\mathcal{M}\left(L_{3}\right)$ does not depend on $y$, and that in addition $\mathcal{M}\left(L_{3}\right)=0$ in the case of Dirichlet conditions. As in (i), we now consider $L_{3}$ as a function of $\mathcal{D}\left(\bar{\Omega}, \mathcal{C}_{\sharp}^{\infty}\left(Y_{1}\right)\right)$; let then $\psi$ be defined by $\psi(x, y)=\int_{-1}^{x_{3}} L_{3}(\hat{x}, t, y) \mathrm{d} t$. With the two conditions above on $L_{3}, \psi$ is an admissible test function such that $\partial_{3} \psi=L_{3}$; with densiry arguments again, this completes the proof of (ii).

Part (iii) is just a restatement of the definition of $\mathbb{M}^{0}$. 
We now give two technical lemmas that are used in the next section to caracterize the limit $\mathbf{M}$.

Lemma 7.2. Let $\left(u^{\varepsilon}\right)_{\varepsilon>0}$ be bounded in $H^{1}(\Omega)$. Let $u \in H^{1}(\Omega)$ and $u^{1} \in L^{2}\left(\Omega ; H_{\sharp}^{1}(Y)\right)$ be functions such that $\left(u^{\varepsilon}\right)_{\varepsilon>0}$ converges weakly to $u$ in $H^{1}(\Omega)$, and $\left(\nabla u^{\varepsilon}\right)_{\varepsilon>0}$ two-scale converges to $\nabla u+\nabla_{y} u^{1}$. Then

$$
\forall v \in \mathcal{D}\left(\Omega ; C_{\sharp}^{\infty}(Y)\right), \quad \lim _{\varepsilon \rightarrow 0} \int_{\Omega} \frac{u^{\varepsilon}}{\varepsilon} \partial_{y_{\alpha}} v^{\varepsilon} \mathrm{d} x=\int_{\Omega \times Y} u^{1} \partial_{y_{\alpha}} v \mathrm{~d} x \mathrm{~d} y .
$$

Proof : One just has to pass to the limit in

$$
\int_{\Omega} \partial_{\alpha} u^{\varepsilon}(x) v(x, \widehat{x} / \varepsilon) \mathrm{d} x=-\int_{\Omega} u^{\varepsilon}(x) \partial_{\alpha} v(x, \widehat{x} / \varepsilon) \mathrm{d} x-\frac{1}{\varepsilon} \int_{\Omega} u^{\varepsilon}(x) \partial_{y_{\alpha}} v(x, \widehat{x} / \varepsilon) \mathrm{d} x .
$$

An integration by parts of the first term on the right-hand side yields then the result.

Lemma 7.3. The family $\left(\frac{\varepsilon^{2}}{a} \int_{\Omega}\left(R_{\alpha 3 k l}^{\varepsilon} K_{k l}^{a}\left(\mathbf{u}^{b}\right)+d_{\alpha 3 k}^{\varepsilon} L_{k}^{a}\left(\varphi^{b}\right)\right) \tilde{v}_{\alpha} \mathrm{d} x\right)$ tends to zero for any $\tilde{v}_{\alpha} \in \mathcal{D}\left(\bar{\omega} ; C_{\sharp}^{\infty}(Y)\right)$ such that $\tilde{v}_{\alpha}=0$ on $\Gamma_{D}$.

Proof : Choose $\mathbf{v}=\varepsilon^{2} x_{3}\left(\tilde{v}_{1}, \tilde{v}_{2}, 0\right)$ and $\psi=0$ in (14), where $\tilde{v}_{\alpha} \in \mathcal{D}\left(\bar{\omega} ; C_{\sharp}^{\infty}(Y)\right)$ and $\tilde{v}_{\alpha}=0$ on $\Gamma_{D}$. Then

$$
\begin{aligned}
\varepsilon^{2} \int_{\Omega}\left(R_{\alpha \beta k l}^{\varepsilon} K_{k l}^{a}\left(\mathbf{u}^{b}\right)+d_{\alpha \beta k}^{\varepsilon} L_{k}^{a}\left(\varphi^{b}\right)\right)\left(s_{\alpha \beta}(\mathbf{v})+\frac{1}{\varepsilon} S_{\alpha \beta}(\mathbf{v})\right) \mathrm{d} x+ \\
\varepsilon^{2} a^{-1} \int_{\Omega} 2\left(R_{\alpha 3 k l}^{\varepsilon} K_{k l}^{a}\left(\mathbf{u}^{b}\right)+d_{\alpha 3 k}^{\varepsilon} L_{k}^{a}\left(\varphi^{b}\right)\right) \tilde{v}_{\alpha} \mathrm{d} x=\varepsilon^{2} \ell_{u}^{b}(\mathbf{v}) .
\end{aligned}
$$

Passing to the limit yields the result.

\subsubsection{Characterization of the limit $M$.}

The first lemma of this section relates to the form of the limit $\mathbf{M}$. We prove that $\mathbf{M} \in \phi_{c}+\mathbb{M}$ where $\phi_{c}=\left(\mathbf{0}_{9}, \varphi_{c}\right)$. The second gives a first variational characterization of $\mathbf{M}$.

Lemma 7.4. Let $\mathbf{M}$ designates the two-scale limit, up to the extraction of a subsequence, of $\left(\mathbf{M}^{b}\left(\mathbf{U}^{b}\right)\right)_{b>0}$. Then $\mathbf{M} \in \phi_{c}+\mathbb{M}$ where $\phi_{c}=\left(\mathbf{0}_{9}, \varphi_{c}\right)$, or in other words:

(i) there exist $\mathbf{u} \in \mathbf{V}_{K L}$ and $\mathbf{u}^{1} \in \mathbf{V}_{K L}^{1}$ such that $K_{a \beta}=s_{\alpha \beta}(\mathbf{u})+S_{\alpha \beta}\left(\mathbf{u}^{1}\right)$;

(ii) $L_{1}=L_{2}=0$;

(iii) $L_{3} \in L^{2}\left(\Omega \times Y_{1}\right), \mathcal{M}\left(L_{3}\right)$ is independant of $y$; and $\mathcal{M}\left(L_{3}\right)=\varphi_{c}$ for Dirichlet conditions.

The third point is just a restatement of Lemma 4.1 (vii). 
Proof of (i): In view of Lemma 4.1, to prove (i), it remains to prove that $\mathbf{u}^{1} \in \mathbf{V}_{K L}^{1}$. The choice $u_{3}^{1}=0$ has been done in Lemma 4.1 (iv). The computation of $u_{\alpha}^{1}$ requires some calculus.

First, with a few integration by parts we get

$$
\begin{aligned}
& \int_{\Omega}\left(\partial_{1} u_{2}^{b}-\partial_{2} u_{1}^{b}\right) \partial_{3} v^{\varepsilon} \mathrm{d} x= \\
& 2 a \int_{\Omega} K_{23}^{a}\left(u^{b}\right)\left(\partial_{1} v^{\varepsilon}+\frac{1}{\varepsilon} \partial_{y_{1}} v^{\varepsilon}\right) \mathrm{d} x-2 a \int_{\Omega} K_{13}^{a}\left(u^{b}\right)\left(\partial_{2} v^{\varepsilon}+\frac{1}{\varepsilon} \partial_{y_{2}} v^{\varepsilon}\right) \mathrm{d} x
\end{aligned}
$$

for all $v \in \mathcal{D}\left(\Omega, \mathcal{C}_{\sharp}^{\infty}(Y)\right)$. Passing to the limit, as $a / \varepsilon$ tends to zero, we get

$$
\forall v \in \mathcal{D}\left(\Omega, \mathcal{C}_{\sharp}^{\infty}(Y)\right), \quad \int_{\Omega \times Y}\left(\partial_{1} u_{2}-\partial_{2} u_{1}\right) \partial_{3} v \mathrm{~d} x \mathrm{~d} y+\int_{\Omega \times Y}\left(\partial_{y_{1}} u_{2}^{1}-\partial_{y_{2}} u_{1}^{1}\right) \partial_{3} v \mathrm{~d} x \mathrm{~d} y=0 .
$$

But because $\mathbf{u} \in \mathbf{V}_{K L}$, this reduces to

$$
\forall v \in \mathcal{D}\left(\Omega, C_{\sharp}^{\infty}(Y)\right), \quad \int_{\Omega \times Y}\left(\partial_{y 1} u_{2}^{1}-\partial_{y_{2}} u_{1}^{1}\right) \partial_{3} v \mathrm{~d} x \mathrm{~d} y=0 .
$$

Now, we show that

$$
\forall v \in \mathcal{D}(\Omega \times Y), \quad \int_{\Omega \times Y} \partial_{y_{\beta}} u_{\alpha}^{1} \partial_{33}^{2} v \mathrm{~d} x \mathrm{~d} y=0
$$

As $\left(K_{33}^{a}\left(\mathbf{u}^{b}\right)\right)$ is bounded, $\varepsilon^{-1} \int_{\Omega} \partial_{3} u_{3}^{b}\left(\partial_{\alpha} \tilde{v}^{\varepsilon}+\varepsilon^{-1} \partial_{y_{\alpha}} \tilde{v}^{\varepsilon}\right) \mathrm{d} x$ tends to zero for any $\tilde{v} \in$ $\mathcal{D}\left(\Omega, \mathcal{C}_{\sharp}^{\infty}(Y)\right)$; hence, $\varepsilon^{-1} \int_{\Omega} \partial_{\alpha} u_{3}^{b} \partial_{3} \tilde{v}^{\varepsilon} \mathrm{d} x$ tends to zero. Also, since $\left(K_{\alpha 3}^{a}\right)$ is bounded, $\varepsilon^{-1} s_{\alpha 3}\left(\mathbf{u}^{b}\right)$ tends to zero. Consequently, $\varepsilon^{-1} \int_{\Omega} \partial_{3} u_{\alpha}^{b} \partial_{3} \tilde{v}^{\varepsilon} \mathrm{d} x$ tends to zero. Choosing now $\tilde{v}=\partial_{y_{\beta}} v$ where $v \in \mathcal{D}(\Omega \times Y)$, one gets that $\varepsilon^{-1} \int_{\Omega} u_{\alpha}^{b} \partial_{y_{\beta}} \partial_{33}^{2} v^{\varepsilon} \mathrm{d} x$ tends to zero. Then, using Lemma 7.2 , we get (42).

Now, we are able to conclude the proof. Equation (42) implies that $\nabla_{y} u_{\alpha}^{1}$ is affine with respect to $x_{3}$. Thus, since $\left(u_{1}^{1}, u_{2}^{1}\right)$ is defined up to a function of $x, u_{\alpha}^{1}(x, y)=\bar{u}_{\alpha}^{1}(\hat{x}, y)+$ $x_{3} u_{\alpha}^{2}(\hat{x}, y)$ where $\bar{u}_{\alpha}^{1}, u_{\alpha}^{2} \in L^{2}(\omega \times Y)$. But then $\partial_{3} u_{\alpha}^{1}=\bar{u}_{\alpha}^{1} \in L^{2}(\omega \times Y)$. On the other hand, in view of $(41),\left(\partial_{3} u_{1}^{1}, \partial_{3} u_{2}^{1}\right)$ is curl free, so that there exists $c_{\alpha} \in L^{2}(\omega)$ and $u_{3}^{2} \in L^{2}\left(\omega ; H_{\sharp}^{1}(Y)\right)$ such that $\partial_{3} u_{\alpha}^{1}=c_{\alpha}-\partial_{y_{\alpha}} u_{3}^{2}$. This finally implies that $\left(u_{1}^{1}, u_{2}^{1}\right)$ may be chosen of the form $u_{\alpha}^{1}=\bar{u}_{\alpha}^{1}-x_{3} \partial_{y_{\alpha}} u_{3}^{2}$ where $\overline{\mathbf{u}}^{1} \in\left(L^{2}(\omega \times Y)\right)^{2}$ and $u_{3}^{2} \in L^{2}\left(\omega ; H_{\sharp}^{1}(Y)\right)$.

Proof of (ii): Passing to the limit in

$$
a \int_{\Omega_{1}^{\varepsilon}} L_{3}^{a}\left(\varphi^{b}\right)\left(\partial_{\alpha} \psi^{\varepsilon}+\frac{1}{\varepsilon} \partial_{y_{\alpha}} \psi^{\varepsilon}\right) \mathrm{d} x=\int_{\Omega_{1}^{\varepsilon}} L_{\alpha}^{a}\left(\varphi^{b}\right) \partial_{3} \psi^{\varepsilon} \mathrm{d} x
$$


because $a / \varepsilon$ tends to zero, we get that

$$
\forall \psi \in \mathcal{D}\left(\Omega \times Y_{1}\right), \int_{\Omega \times Y_{1}} L_{\alpha} \partial_{3} \psi \mathrm{d} x \mathrm{~d} y=0 .
$$

This proves that $L_{1}$ and $L_{2}$ are independent of $x_{3}$. On the other hand, a few integrations by parts, with test functions $\psi$ independent of $x_{3}$, yield

$$
\begin{aligned}
& a \int_{\Omega_{1}^{\varepsilon}} L_{3}^{a}\left(\varphi^{b}\right)\left(x_{3}-1\right)\left(\partial_{\alpha} \psi^{\varepsilon}+\frac{1}{\varepsilon} \partial_{y_{\alpha}} \psi^{\varepsilon}\right) \mathrm{d} x= \\
& \quad \int_{\Omega_{1}^{\varepsilon}} \partial_{\alpha} \varphi^{b} \psi^{\varepsilon} \mathrm{d} x+2 \int_{\omega_{1}^{\varepsilon}}\left(\varphi_{m}^{b}-a \varphi_{c}^{b}\right)\left(\partial_{\alpha} \psi^{\varepsilon}+\frac{1}{\varepsilon} \partial_{y_{\alpha}} \psi^{\varepsilon}\right) \mathrm{d} \hat{x} .
\end{aligned}
$$

The left-hand side tends to zero because $a, \varepsilon$ and $a / \varepsilon$ tend to zero. Since $\varphi_{m}^{b}$ and $\varphi_{c}^{b}$ are constant on each connected part of $\omega_{1}^{\varepsilon}$, the last term on the right is zero. Therefore, passing to the limit, we get

$$
0=2 \int_{\omega \times Y_{1}} L_{\alpha} \psi \mathrm{d} x \mathrm{~d} y .
$$

Since $L_{\alpha}$ does not depend on $x_{3}$, this proves that $L_{\alpha}=0$.

Lemma 7.5 The family $\left(\mathbf{M}^{a}\left(\mathbf{U}^{b}\right)\right)_{b>0}$ two-scale converges to $\mathbf{M}$ which is the unique solution in $\phi_{c}+\mathbb{M}$ of

$$
\left\{\begin{aligned}
\forall \widetilde{\mathbf{M}}=(\widetilde{\mathbf{K}}, \widetilde{\mathbf{L}}) \in \mathbb{M}, \quad \int_{\Omega \times Y} \widetilde{\mathbf{M}} \mathcal{R}{ }^{t} \mathbf{M} \mathrm{d} x \mathrm{~d} y+2 G \int_{\Omega \times Y_{1}} \mathcal{M}\left(L_{3}\right) \mathcal{M}\left(\tilde{L}_{3}\right) \mathrm{d} x \mathrm{~d} y \\
+2 G_{1} \int_{\Omega \times Y_{1}} \partial_{\alpha} \mathcal{M}\left(L_{3}\right) \partial_{\alpha} \mathcal{M}\left(\tilde{L}_{3}\right) \mathrm{d} x \mathrm{~d} y=\ell_{u}(\mathbf{v})+\ell_{\varphi}\left(\tilde{L}_{3}\right)
\end{aligned}\right.
$$

where $\mathbb{M}$ is defined in (38), and

$$
\ell_{\varphi}\left(\tilde{L}_{3}\right)=\int_{\Omega \times Y_{1}} h \tilde{L}_{3} \mathrm{~d} x \mathrm{~d} y=2\left|Y_{1}\right| \int_{\omega} h \mathcal{M}\left(\tilde{L}_{3}\right) \mathrm{d} \hat{x} .
$$

Remark : as usual, the convergence is a priori for a subsequence and the uniqueness of the solution to the limit problem shows a posteriori that the whole family converges.

Proof First, successive multiplications of (14) by $a^{2}, a$ and 1 and passing to the limit yield for all $\mathbf{V} \in \mathbf{W}_{a d}^{1}$

$$
\left\{\begin{array}{l}
\int_{\Omega \times Y} \mathbf{M}^{02}(\mathbf{V}) \mathcal{R}^{t} \mathbf{M} \mathrm{d} x \mathrm{~d} y=0, \\
\int_{\Omega \times Y} \mathbf{M}^{01}(\mathbf{V}) \mathcal{R}^{t} \mathbf{M} \mathrm{d} x \mathrm{~d} y+2 G \int_{\Omega \times Y_{1}} \mathcal{M}\left(L_{3}\right) \mathcal{M}\left(\partial_{3} \psi\right) \mathrm{d} x \mathrm{~d} y \\
\quad+2 G_{1} \int_{\Omega \times Y_{1}} \partial_{\alpha} \mathcal{M}\left(L_{3}\right) \partial_{\alpha} \mathcal{M}\left(\partial_{3} \psi\right) \mathrm{d} x \mathrm{~d} y=\ell_{\varphi}\left(\tilde{L}_{3}\right) \text { if } \mathbf{M}^{02}(\mathbf{V})=\mathbf{M}^{11}(\mathbf{V})=\mathbf{0}, \\
\int_{\Omega \times Y} \mathbf{M}^{00}(\mathbf{V}) \mathcal{R}^{t} \mathbf{M} \mathrm{d} x \mathrm{~d} y=\ell_{u}(\mathbf{v}) \text { if } \mathbf{M}^{02}(\mathbf{V})=\mathbf{M}^{11}(\mathbf{V})=\mathbf{M}^{01}(\mathbf{V})=\mathbf{M}^{10}(\mathbf{V})=\mathbf{0} .
\end{array}\right.
$$


Second, let $\mathbf{v}=\left(\bar{v}_{\alpha}-x_{3} \partial_{y_{\alpha}} v_{3}^{2}, v_{3}^{2}, 0\right) \in \mathbf{V}_{K L}^{1} \cap \mathbf{W}_{a d}^{1}$, with $v_{3}^{2}=0$ in a neighbourhood of $\Gamma_{D}$, and choose

$$
\mathbf{V}^{\varepsilon}=\varepsilon\left(\bar{v}_{1}-x_{3} \partial_{y_{1}} v_{3}^{2}, \bar{v}_{2}-x_{3} \partial_{y_{2}} v_{3}^{2}, \varepsilon v_{3}^{2}, 0\right)
$$

in (14), so that $\varepsilon \mathbf{M}^{00}\left(\mathbf{V}^{\varepsilon}\right)$ tends to $\mathbf{0}, \mathbf{M}^{02}\left(\mathbf{V}^{\varepsilon}\right)=\mathbf{0}$, and

$$
\frac{1}{a} \mathbf{M}^{01}\left(\mathbf{V}^{\varepsilon}\right)+\frac{1}{a \varepsilon} \mathbf{M}^{11}\left(\mathbf{V}^{\varepsilon}\right)=\left(\mathbf{0}_{2 \times 2}, \frac{\varepsilon^{2}}{2 a} \partial_{\alpha} v_{3}^{2}, \mathbf{0}_{4}\right) .
$$

Using lemma 7.3, passing to the limit in (14) yields

$$
\int_{\Omega \times Y} \mathbf{M}^{10}(\mathbf{V}) \mathcal{R}^{t} \mathbf{M} \mathrm{d} x \mathrm{~d} y=0
$$

for any $\mathbf{V}=(\mathbf{v}, 0) \in \mathbf{W}_{a d}^{1}$ with $\mathbf{v} \in \mathbf{V}_{K L}^{1}$. Now, summing the equations (46)-(47) and using then the density lemma 7.1 , we get (44).

Last, uniqueness of the solution to this system is a simple application of Lax-Milgram lemma.

\subsection{Proof of Theorem 5.1.}

From now on, an important use is made of the assumption that $\mathcal{R}$ does not depend on $x_{3}$.

\subsubsection{Proof of Theorem 5.1 : Dirichlet conditions.}

We recall that here $\mathcal{M}\left(L_{3}\right)=\varphi_{c}$ and that $\mathcal{M}$ and $\mathcal{N}$ have been defined in (1). The proof is in two steps. The first one consists in eliminating $K_{i 3}$ and $\mathcal{N}\left(L_{3}\right)$; the second one is for eliminating the $y$-variable. A key for many simplifications is that $\mathcal{R}$ does not depend on $x_{3}$ and so $\mathcal{M}(\mathbf{M})$ and $\mathcal{N}(\mathbf{M})$ bring separate contributions.

First step. Remembering that in the present case, $G=G_{1}=0$ and $h=0$, Equation (44) of Lemma 7.5 reduces to

$$
\forall \widetilde{\mathbf{M}} \in \mathbb{M}, \quad \int_{\Omega \times Y} \widetilde{\mathbf{M}} \mathcal{R}{ }^{t} \mathbf{M} \mathrm{d} x \mathrm{~d} y=\ell_{u}(\mathbf{v})
$$

Splitting $\mathbf{M}$ as $\mathbf{M}=\mathcal{M}(\mathbf{M})+\mathcal{N}(\mathbf{M})$ and taking advantage of the fact that $\mathcal{R}$ does not depend on $x_{3}$, this is equivalent to

$$
\forall \widetilde{\mathbf{M}} \in \mathbb{M}, \quad \int_{\Omega \times Y} \mathcal{M}(\widetilde{\mathbf{M}}) \mathcal{R}^{t} \mathcal{M}(\mathbf{M}) \mathrm{d} x \mathrm{~d} y+\int_{\Omega \times Y} \mathcal{N}(\widetilde{\mathbf{M}}) \mathcal{R}^{t} \mathcal{N}(\mathbf{M}) \mathrm{d} x \mathrm{~d} y=\ell_{u}(\mathbf{v}) .
$$

Let us chose $\widetilde{\mathbf{M}}:=\mathcal{M}(\widetilde{\mathbf{M}})$ as test functions in (48) where $\widetilde{\mathbf{M}} \in \mathbb{M}^{-1} \oplus \mathbb{M}^{-2}$. Because the function $\mathbf{v}$ associated with such a $\widetilde{\mathbf{M}}$ is $\mathbf{v}=\mathbf{0}$, and because $\mathcal{N} \circ \mathcal{M}=0$, we get

$$
\forall \widetilde{\mathbf{M}} \in \mathbb{M}^{-1} \oplus \mathbb{M}^{-2}, \quad \int_{\Omega \times Y} \mathcal{M}(\widetilde{\mathbf{M}}) \mathcal{R}^{t} \mathcal{M}(\mathbf{M}) \mathrm{d} x \mathrm{~d} y=0
$$


Using again that $\mathcal{R}$ is independant of $x_{3}$, this may be rewritten as

$$
\forall \widetilde{\mathbf{M}} \in \mathbb{M}^{-1} \oplus \mathbb{M}^{-2}, \quad \int_{\Omega \times Y} \widetilde{\mathbf{M}} \mathcal{R}^{t} \mathcal{M}(\mathbf{M}) \mathrm{d} x \mathrm{~d} y=0
$$

This holds true in particular for $\widetilde{\mathbf{M}}$ of the form $\widetilde{\mathbf{M}}=\left(\mathbf{0}_{2 \times 2}, \tilde{K}_{i 3}, \mathbf{0}_{3}\right)$ and thus shows that

$$
\Pi_{2} \mathcal{R}{ }^{t} \mathcal{M}(\mathbf{M})=\mathbf{0}
$$

On the other hand, Lemma 7.4 shows that one may decompose $\mathbf{M}$ as

$$
\mathbf{M}=\Pi \mathbf{M}+\mathbf{M}^{0}
$$

where $\Pi \mathbf{M} \in \phi_{c}+\mathbb{M}^{-1} \oplus \mathbb{M}^{-2}, \mathbf{M}^{0} \in \mathbb{M}^{0}$, and that $\mathcal{M}(\Pi \mathbf{M})$ may be written as

$$
\begin{aligned}
\mathcal{M}(\Pi \mathbf{M}) & =\left(\mathbf{0}_{2 \times 2}, \mathcal{M}\left(K_{i 3}\right), \mathbf{0}_{2}, \mathcal{M}\left(L_{3}\right)\right) \\
& =\phi_{c}+\Pi_{2} \mathcal{M}(\mathbf{M}) .
\end{aligned}
$$

Hence, applying $\mathcal{M}$ to (50) we get

$$
\begin{aligned}
\mathcal{M}(\mathbf{M}) & =\phi_{c}+\mathcal{M}\left(\Pi_{2} \mathbf{M}\right)+\mathcal{M}\left(\mathbf{M}^{0}\right) \\
& =\phi_{c}+\Pi_{2} \mathcal{M}\left(\Pi_{2} \mathbf{M}\right)+\mathcal{M}\left(\mathbf{M}^{0}\right) .
\end{aligned}
$$

Applying now $\Pi_{2} \mathcal{R}$ to this identity, with (49), we obtain then

$$
\mathbf{0}=\Pi_{2} \mathcal{R}{ }^{t} \phi_{c}+\left(\Pi_{2} \mathcal{R} \Pi_{2}\right){ }^{t} \mathcal{M}\left(\Pi_{2} \mathbf{M}\right)+\Pi_{2} \mathcal{R}{ }^{t} \mathcal{M}\left(\mathbf{M}^{0}\right),
$$

and therefore

$$
{ }^{t} \mathcal{M}\left(\Pi_{2} \mathbf{M}\right)=\mathbf{T}_{\mathcal{M}}{ }^{t}\left(\phi_{c}+\mathcal{M}\left(\mathbf{M}^{0}\right)\right),
$$

where $\mathbf{T}_{\mathcal{M}}$ has been defined in (22).

Similarly, let us now consider in (48) test functions of the form $\mathcal{N}(\widetilde{\mathbf{M}})$ where $\widetilde{\mathbf{M}}=$ $\left(\mathbf{0}_{4}, \tilde{K}_{i 3}, \mathbf{0}_{2}, \tilde{L}_{3}\right), \tilde{K}_{i 3}, \tilde{L}_{3}$ being given functions in $L^{2}(\Omega \times Y)$ and $L^{2}\left(\Omega \times Y_{1}\right)$ respectively. As $\mathcal{M}\left(\mathcal{N}\left(\tilde{L}_{3}\right)\right)=0, \mathcal{N}(\widetilde{\mathbf{M}}) \in \mathbb{M}^{-1} \oplus \mathbb{M}^{-2}$ so that this choice is correct. Proceding as for the $\mathcal{M}$-component of $\mathbf{M}$, we get that

$$
\forall \widetilde{\mathbf{M}}=\left(\mathbf{0}_{4}, \tilde{K}_{i 3}, \mathbf{0}_{2}, \tilde{L}_{3}\right), \quad \int_{\Omega \times Y} \widetilde{\mathbf{M}} \mathcal{R}^{t} \mathcal{N}(\mathbf{M}) \mathrm{d} x \mathrm{~d} y=0
$$

or in other words, $\Pi \mathcal{R}{ }^{t} \mathcal{N}(\mathbf{M})=\mathbf{0}$. Then, from (50),

$$
\mathbf{0}=\Pi \mathcal{R}{ }^{t} \mathcal{N}(\Pi \mathbf{M})+\Pi \mathcal{R}{ }^{t} \mathcal{N}\left(\mathbf{M}^{0}\right),
$$

and thus,

$$
{ }^{t} \mathcal{N}(\Pi \mathbf{M})=\mathbf{T}_{\mathcal{N}}{ }^{t} \mathcal{N}\left(\mathbf{M}^{0}\right)
$$

where $\mathbf{T}_{\mathcal{N}}$ has been defined in (22). Thus, with (51), (52), (50) and (53) we have 


$$
\mathcal{M}(\mathbf{M})=\left(\mathrm{Id}+\mathbf{T}_{\mathcal{M}}\right){ }^{t}\left(\mathcal{M}\left(\mathbf{M}^{0}\right)+\phi^{c}\right), \text { and } \mathcal{N}(\mathbf{M})=\left(\mathrm{Id}+\mathbf{T}_{\mathcal{N}}\right){ }^{t} \mathcal{N}\left(\mathbf{M}^{0}\right) .
$$

Now, if we choose - in order to maintain a symmetric form to our tensors- in (48) test functions of the special form $\widetilde{\mathbf{M}}=\mathcal{M}\left(\widetilde{\mathbf{M}}^{0}\right)\left(I d+{ }^{t} \mathbf{T}_{\mathcal{M}}\right)+\mathcal{N}\left(\widetilde{\mathbf{M}}^{0}\right)\left(I d+{ }^{t} \mathbf{T}_{\mathcal{N}}\right)$ with $\widetilde{\mathbf{M}}^{0} \in \mathbb{M}^{0}$, taking (54) into account, we get that $\mathbf{M}^{0}$ is the unique solution in $\phi_{c}+\mathbb{M}^{0}$ of

$$
\left\{\begin{array}{c}
\forall \widetilde{\mathbf{M}} \in \mathbb{M}^{0}, \quad \int_{\Omega \times Y}\left(\mathcal{M}(\widetilde{\mathbf{M}}) \mathcal{R}_{\mathcal{M}}{ }^{t} \mathcal{M}\left(\mathbf{M}^{0}\right)+\mathcal{N}(\widetilde{\mathbf{M}}) \mathcal{R}_{\mathcal{N}}{ }^{t} \mathcal{N}\left(\mathbf{M}^{0}\right)\right) \mathrm{d} x \mathrm{~d} y \\
=\ell_{u}(\mathbf{v})-\int_{\Omega \times Y} \mathcal{M}(\widetilde{\mathbf{M}}) \mathcal{R}_{\mathcal{M}}{ }^{t} \phi_{c} \mathrm{~d} x \mathrm{~d} y
\end{array}\right.
$$

where $\mathbf{v}$ is the vector in $\mathbf{V}_{K L}$ associated with $\widetilde{\mathbf{M}}$, and $\mathcal{R}_{\mathcal{M}}$ and $\mathcal{R}_{\mathcal{N}}$ have been defined in (22).

Second step. It remains to eliminate $\mathbf{u}^{1}$. For that purpose, we use the usual arguments of linear homogenization. First note that the definitions (21) of $\mathbf{V}_{K L}$ and (39) of $\mathbf{V}_{K L}^{1}$ imply that

$$
\left\{\begin{array}{l}
\forall \widetilde{\mathbf{M}} \in \mathbb{M}^{0}, \quad \mathcal{M}(\widetilde{\mathbf{M}})=\left(s_{\alpha \beta}(\overline{\mathbf{v}})+S_{\alpha \beta}\left(\overline{\mathbf{v}}^{1}\right), \mathbf{0}_{6}\right) \\
\forall \widetilde{\mathbf{M}} \in \mathbb{M}^{0}, \quad \mathcal{N}(\widetilde{\mathbf{M}})=-x_{3}\left(\partial_{\alpha \beta}^{2} v_{3}+\partial_{y_{\alpha} y_{\beta}}^{2} v_{3}^{2}, \mathbf{0}_{6}\right)
\end{array}\right.
$$

where $\mathbf{v} \in \mathbf{V}_{K L}$ and $\left(\bar{v}_{1}^{1}, \bar{v}_{2}^{1}, v_{3}^{2}\right) \in \mathbf{V}_{K L}^{1}$ are the fields associated with $\widetilde{\mathbf{M}}$. Also, recall that

$$
\mathbf{M}^{0}=\left(\left(s_{\alpha \beta}(\mathbf{u})+\left(S_{\alpha \beta}\left(\mathbf{u}^{1}\right)\right)_{\alpha \beta=1,2}, \mathbf{0}_{6}\right) \text { with } \mathbf{u} \in \mathbf{V}_{K L} \text { and } \mathbf{u}^{1} \in \mathbf{V}_{K L}^{1}\right.
$$

Considering $\mathbf{v}=\mathbf{0}$ in (55), we thus get that for all $\overline{\mathbf{v}}^{1} \in\left(H_{\sharp}^{1}(Y)\right)^{2}$,

$$
\int_{Y} S_{\alpha \beta}\left(\overline{\mathbf{v}}^{1}\right) R_{\mathcal{M} \alpha \beta \lambda \mu} S_{\lambda \mu}\left(\overline{\mathbf{u}}^{1}\right) \mathrm{d} y=-\int_{Y} S_{\alpha \beta}\left(\overline{\mathbf{v}}^{1}\right)\left(R_{\mathcal{M} \alpha \beta \lambda \mu} s_{\lambda \mu}(\overline{\mathbf{u}})+d_{\mathcal{M} 3 \alpha \beta} \varphi_{c}\right) \mathrm{d} y
$$

and for all $v_{3}^{2} \in H_{\sharp}^{2}(Y)$,

$$
\int_{Y} \partial_{y_{\alpha} y_{\beta}}^{2} v_{3}^{2} R_{\mathcal{N} \alpha \beta \lambda \mu} \partial_{y_{\lambda} y_{\mu}}^{2} u_{3}^{2} \mathrm{~d} y=-\int_{Y} \partial_{y_{\alpha} y_{\beta}}^{2} v_{3}^{2} R_{\mathcal{N} \alpha \beta \lambda \mu} \partial_{\lambda \mu}^{2} u_{3} \mathrm{~d} y
$$

almost everywhere in $\omega$.

Equation (57) implies that $\overline{\mathbf{u}}^{1}=\mathbf{u}_{\mathcal{M}}^{\rho \xi} s_{\rho \xi}(\overline{\mathbf{u}})+\mathbf{u}_{\mathcal{M}}^{3} \varphi_{c}$, where the coefficients functions $\mathbf{u}_{\mathcal{M}}^{\rho \xi}$, $\mathbf{u}_{\mathcal{M}}^{3}$ are defined in (24) and (25), and therefore

$$
s_{\lambda \mu}(\overline{\mathbf{u}})+S_{\lambda \mu}\left(\overline{\mathbf{u}}^{1}\right)=\left(\delta_{\lambda \mu, \rho \xi}+S_{\lambda \mu}\left(\mathbf{u}_{\mathcal{M}}^{\rho \xi}\right)\right) s_{\rho \xi}(\overline{\mathbf{u}})+S_{\lambda \mu}\left(\mathbf{u}_{\mathcal{M}}^{3}\right) \varphi_{c} .
$$

This is for the $\mathcal{M}$-part of $\mathbf{M}^{0}$. Similarly, Equation (58) implies that and $u_{3}^{2}=\mathbf{u}_{\mathcal{N}}^{\rho \xi} \partial_{\rho \xi}^{2} u_{3}$ where the $\mathbf{u}_{\mathcal{N}}^{\rho \xi}$ are also defined by $(24)$, and this leads to 


$$
\partial_{\lambda \mu}^{2} u_{3}+\partial_{y_{\lambda} y_{\mu}}^{2} u_{3}^{2}=\left(\delta_{\lambda \mu, \rho \xi}+\partial_{y_{\lambda} y_{\mu}}^{2}\left(\mathbf{u}_{\mathcal{N}}^{\rho \xi}\right)\right) \partial_{\rho \xi}^{2} u_{3}
$$

Now we take in (55) test functions as in (56), with the special forms $\overline{\mathbf{v}}^{1}=\mathbf{u}_{\mathcal{M}}^{\gamma \delta} s_{\gamma \delta}(\overline{\mathbf{v}})$ and $v_{3}^{2}=\mathbf{u}_{\mathcal{N}}^{\gamma \delta} \partial_{\gamma \delta}^{2} v_{3}$. This leads to the system (29) with

$$
\left\{\begin{aligned}
d_{\mathcal{M} 3 \gamma \delta}^{H} & =\int_{Y}\left(\delta_{\alpha \beta, \gamma \delta}+S_{\alpha \beta}\left(\mathbf{u}_{\mathcal{M}}^{\gamma \delta}\right)\right)\left(R_{\mathcal{M} \alpha \beta \lambda \mu} S_{\lambda \mu}\left(\mathbf{u}_{\mathcal{M}}^{3}\right)+d_{\mathcal{M} 3 \alpha \beta}\right) \mathrm{d} y \\
R_{\mathcal{M} \gamma \delta \rho \xi}^{H} & =\int_{Y}\left(\delta_{\alpha \beta, \gamma \delta}+S_{\alpha \beta}\left(\mathbf{u}_{\mathcal{M}}^{\gamma \delta}\right)\right) R_{\mathcal{M} \alpha \beta \lambda \mu}\left(\delta_{\lambda \mu, \rho \xi}+S_{\lambda \mu}\left(\mathbf{u}_{\mathcal{M}}^{\rho \xi}\right)\right) \mathrm{d} y \\
R_{\mathcal{N} \gamma \delta \rho \xi}^{H} & =\int_{Y}\left(\delta_{\alpha \beta, \gamma \delta}+\partial_{y_{\alpha} y_{\beta}}^{2} u_{\mathcal{N}}^{\gamma \delta}\right) R_{\mathcal{N} \alpha \beta \lambda \mu}\left(\delta_{\lambda \mu, \rho \xi}+\partial_{y_{\lambda} y_{\mu}}^{2} u_{\mathcal{N}}^{\rho \xi}\right) \mathrm{d} y
\end{aligned}\right.
$$

which are exactly the tensors announced in formulae (26)-(27). Note in particular that the coefficient 2 appears on the first term on left hand side of (29) because $\int_{-1}^{1} \mathrm{~d} x_{3}=2$ while $\frac{2}{3}=\int_{-1}^{1} x_{3}^{2} \mathrm{~d} x_{3}$ appears on the second term.

\subsubsection{Proof of Theorem 5.1 : nonlocal mixed conditions.}

The proof is very closed to the one for Dirichlet conditions. The main difference is that in the Dirichlet case, $\mathcal{M}\left(L_{3}\right)=\varphi_{c}$ is a given data, while here $\mathcal{M}\left(L_{3}\right)$ is an unknown function which can not be eliminated.

Let us eliminate the other transverse components $K_{\alpha 3}, K_{33}$ and $\mathcal{N}\left(L_{3}\right)$.

The weak formulation (44) of Lemma 7.5, implies now that

$$
\left\{\begin{array}{l}
\forall \widetilde{\mathbf{M}} \in \mathbb{M}, \quad \int_{\Omega \times Y}\left(\mathcal{M}(\widetilde{\mathbf{M}}) \mathcal{R}^{t} \mathcal{M}(\mathbf{M})+\mathcal{N}(\widetilde{\mathbf{M}}) \mathcal{R}^{t} \mathcal{N}(\mathbf{M})\right) \mathrm{d} x \mathrm{~d} y \\
\quad+2 \int_{\Omega \times Y_{1}}\left(G \mathcal{M}\left(L_{3}\right) \mathcal{M}\left(\tilde{L}_{3}\right)+G_{1} \partial_{\alpha} \mathcal{M}\left(L_{3}\right) \partial_{\alpha} \mathcal{M}\left(\tilde{L}_{3}\right)\right) \mathrm{d} x \mathrm{~d} y=\ell_{u}(\mathbf{v})+\ell_{\varphi}\left(\tilde{L}_{3}\right) .
\end{array}\right.
$$

For $\mathcal{M}(\mathbf{M})$, we use test functions such that $\tilde{L}_{3}=0$, as in Section 7.2.1, and thus the computation go on the same way and the result is the same, except that $\varphi_{c}$ is now replaced by $\mathcal{M}\left(L_{3}\right)$. For $\mathcal{N}(\mathbf{M})$, as $\mathcal{M} \circ \mathcal{N}$ and as $\ell_{\varphi}\left(\mathcal{N}\left(\tilde{L}_{3}\right)\right)=0$ (see (45)) the terms in $G, G_{1}$ and $\ell_{\varphi}$ do not play any part as well, and the computation also go as in 7.2.1. We thus get

$$
\mathcal{M}(\mathbf{M})=\left(\mathrm{Id}+\mathbf{T}_{\mathcal{M}}\right)^{t}\left(\mathcal{M}\left(\mathbf{M}^{0}\right)+\Lambda_{3}\right), \text { and } \mathcal{N}(\mathbf{M})=\left(\mathrm{Id}+\mathbf{T}_{\mathcal{N}}{ }^{t} \mathcal{N}\left(\mathbf{M}^{0}\right)\right.
$$


where $\Lambda_{3}=\left(\mathbf{0}_{9}, \mathcal{M}\left(L_{3}\right)\right)$. Then, with a suitable choice of test functions, (59) implies that $\left(\mathbf{M}^{0}, \mathcal{M}\left(L_{3}\right)\right)$ is the unique solution in $\mathbb{M}^{0} \times L^{2}(\omega)$ of

$$
\left\{\begin{aligned}
\forall \widetilde{\mathbf{M}}^{0} \in \mathbb{M}^{0}, \forall \tilde{L}_{3} \in L^{2}(\omega), & \\
& \int_{\Omega \times Y}\left(\left(\mathcal{M}\left(\widetilde{\mathbf{M}}^{0}\right)+\widetilde{\Lambda}_{3}\right) \mathcal{R}_{\mathcal{M}}{ }^{t}\left(\mathcal{M}\left(\mathbf{M}^{0}\right)+\Lambda_{3}\right)+\mathcal{N}\left(\widetilde{\mathbf{M}}^{0}\right) \mathcal{R}_{\mathcal{N}}{ }^{t} \mathcal{N}\left(\mathbf{M}^{0}\right)\right) \mathrm{d} x \mathrm{~d} y \\
& +2 \int_{\Omega \times Y_{1}}\left(G \mathcal{M}\left(L_{3}\right) \tilde{L}_{3}+G_{1} \partial_{\alpha} \mathcal{M}\left(L_{3}\right) \partial_{\alpha} \tilde{L}_{3}\right) \mathrm{d} x \mathrm{~d} y=\ell_{u}(\mathbf{v})+\ell_{\varphi}\left(\tilde{L}_{3}\right)
\end{aligned}\right.
$$

where $\widetilde{\Lambda}_{3}=\left(\mathbf{0}_{9}, \tilde{L}_{3}\right)$ and $\mathbf{v}$ is the vector of $\mathbf{V}_{K L}$ associated with $\widetilde{\mathbf{M}}^{0}$.

To eliminate the local variable $y$, we proceed as in the Dirichlet case, with $\widetilde{\Lambda}_{3}=\left(\mathbf{0}_{9}, \tilde{L}_{3}\right)$ instead of $\phi_{c}$. First, letting $L_{3}^{0}=\mathcal{M}\left(L_{3}\right)$ and considering in (60) test functions such that $\tilde{L}_{3}=0$ and $\overline{\mathbf{v}}=\mathbf{0}$, we get that $\overline{\mathbf{u}}^{1}=\mathbf{u}_{\mathcal{M}}^{\rho \xi} s_{\rho \xi}(\overline{\mathbf{u}})+\mathbf{u}_{\mathcal{M}}^{3} L_{3}^{0}, u_{3}^{2}=\mathbf{u}_{\mathcal{N}}^{\rho \xi} \partial_{\rho \xi}^{2} u_{3}$. Then, remarking that fot all $\widetilde{\mathbf{M}}^{0} \in \mathbb{M}^{0}$ and all $\tilde{L}_{3} \in L^{2}(\omega)$,

$$
\left\{\begin{array}{l}
\left(\mathcal{M}\left(\widetilde{M}^{0}\right)+\widetilde{\Lambda}_{3}\right)=\left(\left(s_{\alpha \beta}(\bar{v})+S_{\alpha \beta}\left(\bar{v}^{1}\right)\right)_{\alpha, \beta=1,2}, 0_{5}, \tilde{L}_{3}\right), \\
\mathcal{N}\left(\widetilde{M}^{0}\right)=-x_{3}\left(\left(\partial_{\alpha \beta}^{2} v_{3}+\partial_{y_{\alpha} y_{\beta}}^{2} v_{3}^{2}\right)_{\alpha, \beta=1,2}, 0_{6}\right)
\end{array}\right.
$$

with a suitable choice of test functions in (60) we obtain the announced model.

Note that the above calculations would be more complicated with non metallized inclusions, as $\mathcal{M}\left(L_{3}\right)$ would then depend on $y$.

\subsubsection{Proof of Theorem 5.1 : local mixed conditions.}

Here $G_{1}=0$ so that the effective equations simplifies into

$$
\left\{\begin{aligned}
\int_{\omega}\left(2\left(s_{\alpha \beta}(\overline{\mathbf{v}}), \tilde{L}_{3}\right)\left(\begin{array}{cc}
R_{\mathcal{M} \alpha \beta \gamma \delta}^{H} & d_{\mathcal{M} 3 \alpha \beta}^{H} \\
e_{\mathcal{M} 3 \gamma \delta}^{H} & c_{\mathcal{M} 33}^{H}+2\left|Y_{1}\right| G
\end{array}\right)\left(\begin{array}{c}
s_{\gamma \delta}(\overline{\mathbf{u}}) \\
L_{3}^{0}
\end{array}\right)\right) \mathrm{d} \hat{x} \\
+\frac{2}{3} \int_{\omega} \partial_{\alpha \beta}^{2} v_{3} R_{\mathcal{N} \alpha \beta \gamma \delta}^{H} \partial_{\gamma \delta}^{2} u_{3} \mathrm{~d} \hat{x}=\ell_{u}(\mathbf{v})+2\left|Y_{1}\right| \int_{\omega} \tilde{L}_{3} h \mathrm{~d} \hat{x} .
\end{aligned}\right.
$$

This simplification allows to eliminate the unknown $L_{3}^{0}$ that can be computed explicitly in terms of $s_{\gamma \delta}(\overline{\mathbf{u}})$. We choose $\mathbf{v}=\mathbf{0}$ then

$$
\forall \tilde{L}_{3} \in L^{2}(\omega), \int_{\omega}\left(e_{\mathcal{M} 3 \gamma \delta}^{H} s_{\gamma \delta}(\overline{\mathbf{u}})+\left(c_{\mathcal{M} 33}^{H}+2\left|Y_{1}\right| G\right) L_{3}^{0}-\left|Y_{1}\right| h\right) \tilde{L}_{3} \mathrm{~d} \hat{x}=0
$$

so that

$$
\left(c_{\mathcal{M} 33}^{H}+2\left|Y_{1}\right| G\right) L_{3}^{0}=\left|Y_{1}\right| h-e_{\mathcal{M} 3 \gamma \delta}^{H} s_{\gamma \delta}(\overline{\mathbf{u}}) \text { a.e. in } \omega .
$$

Now replacing $L_{3}^{0}$ and restricting ourselves to test functions with $\tilde{L}_{3}=0$, we get the announced model, and thus conclude the proof. 


\section{Proof of Theorem 6.1.}

This section is devoted to the derivation of Theorem 6.1. The proof is based on the general results of Lemma 4.1. As homogenization and plate theory act here on the same level, the proof is in two steps only: first, characterization of the limit $\mathbf{M}$ defined in Lemma 4.1; this is the aim of Section 8.1; second, elimination of the local variable $\left(y_{1}, y_{2}, x_{3}\right)$; this is achieved in Section 8.2.

\subsection{Step 1: Characterization of the limit M.}

\subsubsection{Characterization of $\mathrm{M}$.}

Lemma 8.1. Let $\mathbf{M}=(\mathbf{K}, \mathbf{L})$ be the limit, up to a subsequence, of $\left(\mathbf{M}^{a}\left(\mathbf{U}^{b}\right)\right)$, then (i) there exist $\mathbf{u} \in \mathbf{V}_{K L}$ and $\hat{\mathbf{u}}^{1} \in\left(L^{2}\left(\Omega ; H_{\sharp}^{1}(Y)\right) \cap L^{2}\left(\omega ; H^{1}(Z)\right)^{3}\right.$ such that

$$
\begin{aligned}
& \forall \alpha, \beta \in\{1,2\}, K_{a \beta}=s_{\alpha \beta}(\mathbf{u})+S_{\alpha \beta}\left(\hat{\mathbf{u}}^{1}\right), \\
& \forall i \in\{1,2,3\}, K_{i 3}=S_{i 3}\left(\hat{\mathbf{u}}^{1}\right)
\end{aligned}
$$

(ii) there exists $\varphi^{1} \in L^{2}\left(\omega ; H^{1}\left(Z_{1}\right)\right)$ such that $L=\nabla_{z} \varphi^{1}$;

(iii) in the case of Dirichlet conditions, one may chose $\varphi^{1}$ so that $\varphi^{1}=x_{3} \varphi_{c}+\hat{\varphi}^{1}$ with $\hat{\varphi}^{1} \in L^{2}\left(\omega ; H^{1}\left(Z_{1}\right)\right)$ and $\hat{\varphi}^{1}=0$ on $\Gamma^{+} \cup \Gamma^{-}$;

(iv) in the case of mixed conditions one may chose $\varphi^{1}$ so that $\varphi^{1}=\left(1+x_{3}\right) \mathcal{M}\left(L_{3}\right)+\hat{\varphi}^{1}$ with $\hat{\varphi}^{1} \in L^{2}\left(\omega ; H^{1}\left(Z_{1}\right)\right)$ and $\hat{\varphi}^{1}=0$ on $\Gamma^{+} \cup \Gamma^{-}$.

Proof : First, from Lemma 4.1 we know that there is $\left(\mathbf{u}, \mathbf{u}^{1}\right) \in V_{K L} \times L^{2}\left(\Omega ; \mathbf{H}_{\sharp}^{1}(Y) / \mathbb{R}\right)^{2}$ such that $K_{\alpha \beta}=s_{\alpha \beta}(\mathbf{u})+S_{\alpha \beta}\left(\mathbf{u}^{1}\right)$. Then here, passing to the limit in (40) as $a / \varepsilon \rightarrow 1$, we get, taking $\mathbf{u} \in V_{K L}$ into account, that for all $v \in \mathcal{D}\left(\Omega ; \mathcal{C}_{\sharp}^{\infty}(Y)\right)$,

$$
\int_{\Omega \times Y}\left(\partial_{y_{1}} u_{2}^{1}-\partial_{y_{2}} u_{1}^{1}\right) \partial_{3} v \mathrm{~d} x \mathrm{~d} y=2 \int_{\Omega \times Y}\left(K_{23} \partial_{y_{1}} v-K_{13} \partial_{y_{2}} v\right) \mathrm{d} x \mathrm{~d} y
$$

Hence, see for instance [24, Theorem 5], there is $u_{3}^{2}$ and $c_{1}, c_{2}$ not depending on $y$ such that for $\alpha=1,2, \partial_{3} u_{\alpha}^{1}-2 K_{\alpha 3}=c_{\alpha}-\partial_{y_{\alpha}} u_{3}^{2}$. Also, note that $u_{3}^{2}$ is defined up to the addition of a function of $x$. Here, as $\mathbf{u}^{1}$ is also defined up to the addition of a function of $x$, one may choose $u_{\alpha}^{1}$ so that $c_{\alpha}=0$. Letting $\hat{\mathbf{u}}^{1}=\left(u_{1}^{1}, u_{2}^{2}, u_{3}^{2}\right)$, then $K_{\alpha 3}=S_{\alpha 3}\left(\widehat{\mathbf{u}}^{1}\right)$.

We now prove that $K_{33}=\partial_{3} u_{3}^{2}$. With a few integrations by parts, one easily gets that for any $v \in \mathcal{D}(\Omega \times Y)$,

$$
\begin{aligned}
\int_{\Omega} \partial_{\alpha} u_{\beta}^{b} \partial_{33}^{2} v^{\varepsilon} \mathrm{d} x+\int_{\Omega} \partial_{\beta} u_{3}^{b}\left(\partial_{\alpha 3}^{2} v^{\varepsilon}+\frac{1}{\varepsilon} \partial_{3 y_{\alpha}}^{2} v^{\varepsilon}\right) \mathrm{d} x= \\
2 a \int_{\Omega} K_{\beta 3}^{a}\left(\mathbf{u}^{b}\right) \partial_{\alpha 3}^{2} v^{\varepsilon} \mathrm{d} x+2 \int_{\Omega} K_{\beta 3}^{a}\left(\mathbf{u}^{b}\right) \partial_{3 y_{\alpha}}^{2} v^{\varepsilon} \mathrm{d} x .
\end{aligned}
$$


Besides, from Lemma 4.1, we know that $\left(a^{2} K_{33}^{a}\left(\mathbf{u}^{b}\right)\right)$ and $\left(a K_{33}^{a}\left(\mathbf{u}^{b}\right)\right)$ tend to zero and that $\left(K_{33}^{a}\left(\mathbf{u}^{b}\right)\right)$ tends to $K_{33}$. Thus, integrating by parts again (for the second term on the left hand side) and passing to the limit, we get

$$
\int_{\Omega \times Y}\left(\partial_{\alpha} u_{\beta}+\partial_{y_{\alpha}} u_{\beta}^{1}\right) \partial_{33}^{2} v \mathrm{~d} x \mathrm{~d} y+\int_{\Omega \times Y} K_{33} \partial_{y_{\alpha} y_{\beta}}^{2} v \mathrm{~d} x \mathrm{~d} y=2 \int_{\Omega \times Y} K_{\beta 3} \partial_{y_{\alpha} 3}^{2} v \mathrm{~d} x \mathrm{~d} y
$$

for all $v \in \mathcal{D}(\Omega \times Y)$. But because $\mathbf{u} \in V_{K L}$, this is equivalent to

$$
\int_{\Omega \times Y} \partial_{y_{\alpha}} u_{\beta}^{1} \partial_{33}^{2} v \mathrm{~d} x \mathrm{~d} y+\int_{\Omega \times Y} K_{33} \partial_{y_{\alpha} y_{\beta}}^{2} v \mathrm{~d} x \mathrm{~d} y=2 \int_{\Omega \times Y} K_{\beta 3} \partial_{3 y_{\alpha}}^{2} v \mathrm{~d} x \mathrm{~d} y .
$$

Choosing $v=w_{\alpha}$ in (62), summing for $\alpha=1$ to 2 and using the fact that for each $v$ in $\mathcal{D}(\Omega \times Y)$, there is a $\mathbf{w}$ in $(\mathcal{D}(\Omega \times Y))^{2}$ such that $d_{i v} \mathbf{w}=v$, (62) implies that $\partial_{33}^{2} u_{\beta}^{1}+\partial_{y_{\beta}} K_{33}-2 \partial_{3} K_{\beta 3}=0$ for $\beta=1,2$. As $2 K_{\beta 3}=\partial_{3} u_{\beta}^{1}+\partial_{y_{\beta}} u_{3}^{2}$, this is in turn equivalent to $\partial_{y_{\beta}}\left(\partial_{3} u_{3}^{2}-K_{33}\right)=0$. But because $u_{3}^{2}$ is defined up to an additive function of $x$ we may choose $u_{3}^{2}$ such that $K_{33}=\partial_{3} u_{3}^{2}$. Then $S_{i j}\left(\hat{\mathbf{u}}^{1}\right) \in L^{2}(\Omega \times Y)$ for each pair $(i, j) \in\{1 . .3\}^{2}$ and therefore $\hat{\mathbf{u}}^{1} \in \mathbf{L}^{2}\left(\omega ; H^{1}(Z)\right)$. Last, using (61), $u_{3}^{2}$ is $Y$-periodic. This ends the proof of point (i).

Now, we prove (ii) and (iii) in the case of Dirichlet conditions. From Lemma 4.1, we already know that there exists $\varphi^{1}$ such that $L_{1}=\partial_{y_{1}} \varphi^{1}$ and $L_{2}=\partial_{y_{2}} \varphi^{1}$. Besides, passing to the limit in (43) yields

$$
\int_{\Omega \times Y_{1}} L_{3} \partial_{y_{\alpha}} \psi \mathrm{d} x \mathrm{~d} y=\int_{\Omega \times Y_{1}} \partial_{y_{\alpha}} \varphi^{1} \partial_{3} \psi \mathrm{d} x \mathrm{~d} y
$$

for all $\alpha=1,2$ and all $\psi \in \mathcal{D}\left(\Omega \times Y_{1}\right)$. Thus, $\partial_{3} \varphi^{1}-L_{3}$ does not depend on $y$ in $Y_{1}$. Hence, since $\varphi^{1}$ is defined up to a function of $x$, one may choose $\varphi^{1}$ so that $L_{3}=\partial_{3} \varphi^{1}$ and we have in addition that $\varphi^{1} \in L^{2}\left(\omega ; H^{1}\left(Z_{1}\right)\right)$, so (ii) is proven. On the other hand, as we know from Lemma 4.1 that $\mathcal{M}\left(L_{3}\right)=\varphi_{c}$, we have that $\varphi_{/ \Gamma^{+}}^{1}-\varphi_{/ \Gamma^{-}}^{1}=2 \varphi_{c}$. Since so far, $\varphi^{1}$ is still defined up to a function of $\hat{x}$, we may choose $\varphi^{1}$ so that $\varphi^{1}=\varphi_{c}$ on $\Gamma^{+}$and $\varphi^{1}=-\varphi_{c}$ on $\Gamma^{-}$, or in other words, choose $\varphi^{1}$ as stated in (iii).

The case of mixed conditions is more complicated. Let us reconsider the limit of $\left(\mathbf{L}^{a}\left(\varphi^{b}\right)\right)$ globally. We remark that $\partial_{3} \varphi^{b}=\partial_{3}\left(\varphi^{b}-\varphi_{m}^{b}\right)$, and also, due to the assumption of metallization, that $\partial_{\alpha} \varphi^{b}=\partial_{\alpha}\left(\varphi^{b}-\varphi_{m}^{b}\right)$ for $\alpha=1,2$. The limit $\mathbf{L}$ of $\left(\mathbf{L}^{a}\left(\varphi^{b}\right)\right)$ is therefore also the limit of $\left(\mathbf{L}^{a}\left(\varphi^{b}-\varphi_{m}^{b}\right)\right)$. Besides, as

$$
\varepsilon^{-1} \partial_{3}\left(\varphi^{b}-\varphi_{m}^{b}\right)=\frac{a}{\varepsilon} L_{3}^{a}\left(\varphi^{b}-\varphi_{m}^{b}\right)=\frac{a}{\varepsilon} L_{3}^{a}\left(\varphi^{b}\right),
$$

and as $\varepsilon / a \rightarrow 1,\left(\varepsilon^{-1} \partial_{3}\left(\varphi^{b}-\varphi_{m}^{b}\right)\right)$ is bounded in $L^{2}\left(\Omega_{1}^{\varepsilon}\right)$ and also two-scale converge to $L_{3}$. Thus, if we pose

$$
\tilde{\mathbf{L}}^{b}=\left(\partial_{\alpha}\left(\varphi^{b}-\varphi_{m}\right), \varepsilon^{-1} \partial_{3}\left(\varphi^{b}-\varphi_{m}\right)\right)
$$

then $\left(\tilde{\mathbf{L}}^{b}\right)$ two-scale converges to $\mathbf{L}$. 
Also, by Poincaré inequality, the fact that $\left(\varepsilon^{-1} \partial_{3}\left(\varphi^{b}-\varphi_{m}^{b}\right)\right)$ is bounded in $L^{2}\left(\Omega_{1}^{\varepsilon}\right)$ and that $\varphi^{b}-\varphi_{m}^{b}$ vanishes on $\Gamma_{1}^{-}$implies that $\left(\varepsilon^{-1}\left(\varphi^{b}-\varphi_{m}^{b}\right)\right)$ is bounded in $L^{2}\left(\omega_{1}^{\varepsilon} ; H^{1}(-1,1)\right)$. Hence, there exists $\varphi^{1} \in L^{2}\left(\omega \times Y_{1} ; H^{1}(-1,1)\right)$ such that $\left(\varepsilon^{-1}\left(\varphi^{b}-\varphi_{m}^{b}\right)\right)$ two-scale converges to $\varphi^{1}$, and $\left(\varepsilon^{-1} \partial_{3}\left(\varphi^{b}-\varphi_{m}^{b}\right)\right)$ two-scale converges to $\partial_{3} \varphi^{1}$. Then, passing to the limit in

$$
\begin{aligned}
\int_{\Omega_{1}^{\varepsilon}} \tilde{\mathbf{L}}^{b} \psi^{\varepsilon} \mathrm{d} x= & -\int_{\Omega_{1}^{\varepsilon}}\left(\varphi^{b}-\varphi_{m}\right)\left(\partial_{1} \psi_{1}^{\varepsilon}+\partial_{2} \psi_{2}^{\varepsilon}\right) \mathrm{d} x \\
& -\frac{1}{\varepsilon} \int_{\Omega_{1}^{\varepsilon}}\left(\varphi^{b}-\varphi_{m}\right) \operatorname{div}{ }_{z} \psi^{\varepsilon} \mathrm{d} x+\int_{\Gamma_{1}^{\varepsilon}}\left(\varphi^{b}-\varphi_{m}\right) \psi^{\varepsilon} \cdot \mathbf{n} \mathrm{d} \sigma(x),
\end{aligned}
$$

we get that for any function $\psi$ in $D\left(\Omega \times Y_{1}\right)$,

$$
\int_{\Omega \times Y_{1}} \mathbf{L} \psi \mathrm{d} x \mathrm{~d} y=-\int_{\Omega \times Y_{1}} \varphi^{1} \operatorname{div}_{z} \psi \mathrm{d} x \mathrm{~d} y .
$$

This proves that $\varphi^{1}$ actually belongs to $L^{2}\left(\omega ; H^{1}\left(Y_{1} \times\right]-1,1[)\right)$ and that $\mathbf{L}=\nabla_{z} \varphi^{1}$.

Moreover, the continuity of the trace function implies that $\varphi^{1}=0$ on $\Gamma^{-}$and, with the assumption of metallization, that $\varphi^{1}$ does not depend on $y$ on $\Gamma^{+}$. Then passing to the limit in

$$
\forall \psi \in D\left(\omega \times Y_{1}\right), \frac{a}{\varepsilon} \int_{\Omega_{1}^{\varepsilon}} L_{3}^{a}\left(\varphi^{b}\right) \psi^{\varepsilon} \mathrm{d} x=\frac{1}{2} \int_{\omega_{1}^{\varepsilon}} \operatorname{tr}_{\Gamma^{+}}\left(\frac{\varphi^{b}-\varphi_{m}^{b}}{\varepsilon}\right) \psi^{\varepsilon} \mathrm{d} \hat{x}
$$

we get that $\varphi^{1}=2 \mathcal{M}\left(L_{3}\right)$ on $\Gamma^{+}$. Thus, letting $\hat{\varphi}^{1}=\varphi^{1}-\left(1+x_{3}\right) \mathcal{M}\left(L_{3}\right)$, we get the announced result.

8.1.2 Intermediate limit model. We state now a first limit model, including both global and local variables, that is directly deduced from the convergence results of Lemma 4.1 and the characterization in Lemma 8.1.

The limit $\mathbf{M}$ of $\left(\mathbf{M}^{a}\left(\mathbf{U}^{b}\right)\right)$ is completely determined by the macroscopic fields $\mathbf{U}=\left(\mathbf{u}, \mathcal{M}\left(L_{3}\right)\right)$ $\in\left(\mathbf{0}_{3}, \varphi_{c}\right)+\mathbf{W}^{0}$, where we have let

$$
\begin{aligned}
& \mathbf{W}^{0}=\mathbf{V}_{K L} \times\{0\} \text { in the case of Dirichlet conditions, } \\
& \mathbf{W}^{0}=\mathbf{V}_{K L} \times L^{2}(\omega) \text { otherwise, }
\end{aligned}
$$

and the microscopic fields $\mathbf{U}^{1}=\left(\hat{\mathbf{u}}^{1}, \hat{\varphi}^{1}\right) \in \mathbf{L}^{2}\left(\omega ; \mathbf{W}^{1}\right)$ where $\mathbf{W}^{1}$ is defined in (30). More specifically, since $\partial_{y_{\alpha}} \hat{\varphi}^{1}=\partial_{y_{\alpha}} \varphi^{1}$, the limit $\mathbf{M}$ takes the form $\mathbf{M}=\mathbf{M}^{0}(\mathbf{U})+\mathbf{M}^{1}\left(\mathbf{U}^{1}\right)$ where

$$
\mathbf{M}^{0}(\mathbf{U})=\left(\left(s_{\alpha \beta}(\mathbf{u})\right)_{\alpha, \beta=1,2}, \mathbf{0}_{5}, \mathcal{M}\left(L_{3}\right)\right),
$$

and the operator $\mathbf{M}^{1}$ has been defined in (31).

Now, we state the following intermediate limit model using the definitions (28), (45) of $\ell_{u}, \ell_{\varphi}$ and $L_{3}^{0}=\mathcal{M}\left(L_{3}\right)$. 
Lemma 8.2. The limit $\mathbf{M}$ of $\mathbf{M}^{a}\left(\mathbf{U}^{b}\right)$ takes the form $\mathbf{M}=\mathbf{M}^{0}(\mathbf{U})+\mathbf{M}^{1}\left(\mathbf{U}^{1}\right)$ where $\mathbf{U}=\left(\mathbf{u}, L_{3}^{0}\right) \in\left(\mathbf{0}_{3}, \varphi_{c}\right)+\mathbf{W}^{0}$ and $\mathbf{U}^{1}=\left(\hat{\mathbf{u}}^{1}, \hat{\varphi}^{1}\right) \in \mathbf{L}^{2}\left(\omega ; \mathbf{W}^{1}\right)$ is the unique solution of this form of

$$
\left\{\begin{array}{l}
\forall \mathbf{V}=\left(\mathbf{v}, \widetilde{L}_{3}\right) \in \mathbf{W}^{0}, \forall \mathbf{V}^{1}=\left(\widehat{\mathbf{v}}^{1}, \psi^{1}\right) \in \mathbf{L}^{2}\left(\omega ; \mathbf{W}^{1}\right) \\
\int_{\Omega \times Y}\left(\mathbf{M}^{0}(\mathbf{V})+\mathbf{M}^{1}\left(\mathbf{V}^{1}\right)\right) \mathcal{R}^{t}\left(\mathbf{M}^{0}(\mathbf{U})+\mathbf{M}^{1}\left(\mathbf{U}^{1}\right)\right) \mathrm{d} x \mathrm{~d} y+ \\
4\left|Y_{1}\right| \int_{\omega}\left(G \widetilde{L}_{3} L_{3}^{0}+G_{1} \partial_{\alpha} \widetilde{L}_{3} \partial_{\alpha} L_{3}^{0}\right) \mathrm{d} \hat{x}=l_{u}(\mathbf{v})+\ell_{\varphi}\left(\widetilde{L}_{3}\right) .
\end{array}\right.
$$

Proof: Using the definitions (35) of $\mathbf{W}_{a d}^{1}$ and the notations (36)-(37), multiplying (14) successively by $\varepsilon^{2}, \varepsilon$ and 1 , and passing to the limit, one gets

$$
\left\{\begin{array}{r}
\forall \mathbf{V}^{1} \in \mathbf{W}_{a d}^{1}, \quad \int_{\Omega \times Y}\left(\mathbf{M}^{11}\left(\mathbf{V}^{1}\right)+\mathbf{M}^{02}\left(\mathbf{V}^{1}\right)\right) \mathcal{R}^{t} \mathbf{M} \mathrm{d} x \mathrm{~d} y=0 \\
\forall \mathbf{V}^{1} \in \mathbf{W}_{a d}^{1} \text { with } v_{3}^{1}=0, \quad \int_{\Omega \times Y}\left(\mathbf{M}^{01}\left(\mathbf{V}^{1}\right)+\mathbf{M}^{10}\left(\mathbf{V}^{1}\right)\right) \mathcal{R}^{t} \mathbf{M} \mathrm{d} x \mathrm{~d} y \\
\quad+2 \int_{\Omega \times Y_{1}}\left(G \mathcal{M}\left(\widetilde{L}_{3}\right) L_{3}^{0}+G_{1} \partial_{\alpha} \mathcal{M}\left(\widetilde{L}_{3}\right) \partial_{\alpha} L_{3}^{0}\right) \mathrm{d} x \mathrm{~d} y=\ell_{\varphi}\left(\widetilde{L}_{3}\right) \\
\forall \mathbf{v} \in \mathbf{V}_{K L}, \quad \int_{\Omega \times Y}{ }^{t} \mathbf{M}^{00}((\mathbf{v}, 0)) \mathcal{R}^{t} \mathbf{M} \mathrm{d} x \mathrm{~d} y=\ell_{u}(\mathbf{v}) .
\end{array}\right.
$$

Unlike the preceding models, the first two equations are coupled. Consequently, the computation of $K_{33}$ on one hand and of $\left(K_{13}, K_{23}\right)$ on the other hand cannot be carried out independently. Summing up the three equations above and using usual density results, and restricting ourselves to test functions such that $\widetilde{L}_{3}$ does not depend on $x_{3}$, we get the announced weak formulation. Uniqueness of the solution follows from Lax-Milgram Lemma, and the convergence of the whole family from the uniqueness of the solution.

8.2 Step 2: Derivation of the models. In this section, we complete the proof of Theorem 6.1 by eliminating the local variable $z$ and the corresponding unknown $\mathbf{U}^{1}$. We use notations already introduced in Section 6.1.

For $\mathbf{W}^{1}$ defined in (30), considering (63) with $\mathbf{V}=\mathbf{0}$, we easily get

$$
\left\{\begin{array}{l}
\forall \mathbf{V}^{1}=\left(\mathbf{v}^{1}, \psi^{1}\right) \in \mathbf{W}^{1} \\
\int_{Z} \mathbf{M}^{1}\left(\mathbf{V}^{1}\right) \mathcal{R}{ }^{t} \mathbf{M}^{1}\left(\mathbf{U}^{1}\right) \mathrm{d} z=-\int_{Z} \mathbf{M}^{1}\left(\mathbf{V}^{1}\right) \mathcal{R}{ }^{t} \mathbf{M}^{0}(\mathbf{U}) \mathrm{d} z
\end{array}\right.
$$

Let then $\mathbf{M}_{\mathcal{M}}^{0}(\mathbf{U})=\left(\left(s_{\gamma \delta}(\overline{\mathbf{u}})\right)_{\gamma, \delta=1,2}, \mathbf{0}_{5}, L_{3}^{0}\right)$ and $\mathbf{M}_{\mathcal{N}}^{0}(\mathbf{U})=\left(-x_{3}\left(\partial_{\gamma \delta}^{2} u_{3}\right)_{\gamma, \delta=1,2}, \mathbf{0}_{6}\right)$, where $L_{3}^{0}=\varphi_{c}$ for Dirichlet conditions, then (64) may be rewritten as

$$
\left\{\begin{array}{l}
\forall \mathbf{V}^{1}=\left(\mathbf{v}^{1}, \psi^{1}\right) \in \mathbf{W}^{1} \\
\int_{Z} \mathbf{M}^{1}\left(\mathbf{V}^{1}\right) \mathcal{R}{ }^{t} \mathbf{M}^{1}\left(\mathbf{U}^{1}\right) \mathrm{d} z=-\int_{Z} \mathbf{M}^{1}\left(\mathbf{V}^{1}\right) \mathcal{R}{ }^{t}\left(\mathbf{M}_{\mathcal{M}}^{0}(\mathbf{U})+\mathbf{M}_{\mathcal{N}}^{0}(\mathbf{U})\right) \mathrm{d} z
\end{array}\right.
$$


This proves that

$$
\mathbf{U}^{1}=\mathbf{U}_{\mathcal{M}}^{\lambda \mu} s_{\lambda \mu}(\overline{\mathbf{u}})+\mathbf{U}_{\mathcal{N}}^{\lambda \mu} \partial_{\lambda \mu}^{2} u_{3}+\mathbf{U}^{3} L_{3}^{0}
$$

where $\mathbf{U}_{\mathcal{M}}^{\lambda \mu}, \mathbf{U}_{\mathcal{N}}^{\lambda \mu}$ and $\mathbf{U}^{3}$ have been defined in (32)-(33)-(34). Moreover,

$$
\mathbf{M}^{1}\left(\mathbf{U}^{1}\right)=\mathbf{M}^{1}\left(\mathbf{U}_{\mathcal{M}}^{\lambda \mu}\right) s_{\lambda \mu}(\overline{\mathbf{u}})+\mathbf{M}^{1}\left(\mathbf{U}_{\mathcal{N}}^{\lambda \mu}\right) \partial_{\lambda \mu}^{2} u_{3}+\mathbf{M}^{1}\left(\mathbf{U}^{3}\right) L_{3}^{0},
$$

which implies that

$$
\begin{aligned}
\mathbf{M}^{0}(\mathbf{U}) & +\mathbf{M}^{1}\left(\mathbf{U}^{1}\right)=\left(\mathbf{E}^{\lambda \mu}+\mathbf{M}^{1}\left(\mathbf{U}_{\mathcal{M}}^{\lambda \mu}\right)\right) s_{\lambda \mu}(\overline{\mathbf{u}}) \\
& +\left(-x_{3} \mathbf{E}^{\lambda \mu}+\mathbf{M}^{1}\left(\mathbf{U}_{\mathcal{N}}^{\lambda \mu}\right)\right) \partial_{\lambda \mu}^{2} u_{3}+\left(\left(\mathbf{0}_{9}, 1\right)+\mathbf{M}^{1}\left(\mathbf{U}^{3}\right)\right) L_{3}^{0} .
\end{aligned}
$$

The choice in $(63)$ of $\mathbf{V}^{1}=\left(\widehat{\mathbf{v}}^{1}, \psi^{1}\right) \in \mathbf{W}^{1}$ such that

$$
\mathbf{M}^{1}\left(\mathbf{V}^{1}\right)=\mathbf{M}^{1}\left(\mathbf{u}_{\mathcal{M}}^{\alpha \beta}, \varphi_{\mathcal{M}}^{\alpha \beta}\right) s_{\alpha \beta}(\overline{\mathbf{v}})+\mathbf{M}^{1}\left(\mathbf{u}_{\mathcal{N}}^{\alpha \beta}, \varphi_{\mathcal{N}}^{\alpha \beta}\right) \partial_{\alpha \beta}^{2} v_{3}
$$

in the case of Dirichlet conditions and

$$
\mathbf{M}^{1}\left(\mathbf{V}^{1}\right)=\mathbf{M}^{1}\left(\mathbf{u}_{\mathcal{M}}^{\alpha \beta}, \varphi_{\mathcal{M}}^{\alpha \beta}\right) s_{\alpha \beta}(\overline{\mathbf{v}})+\mathbf{M}^{1}\left(\mathbf{u}_{\mathcal{N}}^{\alpha \beta}, \varphi_{\mathcal{N}}^{\alpha \beta}\right) \partial_{\alpha \beta}^{2} v_{3}+\mathbf{M}^{1}\left(\mathbf{u}_{\mathcal{M}}^{3}, \varphi_{\mathcal{M}}^{3}\right) \widetilde{L}_{3}^{0}
$$

in the case of mixed conditions, where $\left(\mathbf{v}, \widetilde{L}_{3}\right)$ belongs to $\mathbf{V}_{K L} \times\{0\}$ for Dirichlet conditions, to $\mathbf{V}_{K L} \times L^{2}(\omega)$ for local mixed conditions, and $\mathbf{V}_{K L} \times H^{1}(\omega)$ for nonlocal mixed conditions, completes the proof. 


\section{References}

1. Allaire G. Homogenization and two scale-convergence. SIAM J. of Math. An. 1992; 23 (26); 1482-1518.

2. Bensoussan A, Lions J-L, Papanicolaou G. Asymptotic Analysis for Periodic Structures. North Holland, Amsterdam; 1978.

3. Brassart M., Lenczner M. A two-scale model for the periodic homogenization of the wave equation. Journal de Mathématiques Pures et Appliquées 2010; 93(5), 474-517.

4. Caillerie D. Thin elastic and periodic plates. Math. Meth. in Applied Sciences 1984; 6; 159-191.

5. Canon É, Lenczner M. Models of elastic plates with piezoelectric inclusions, part I: models without homogenization. Math. Comp. Model. 1997; 26 (5); 79-106.

6. Canon É, Lenczner M. Deux modèles de plaques minces avec inclusions piézoélectriques et circuits électroniques distribués. C.R. Acad. Sci. Paris, Série II 1998; 326; 793-798.

7. Canon É, Lenczner M. Modelling of thin elastic plates with small piezoelectric inclusions and distributed electronic circuits. Models for inclusions that are small with respect to the thickness of the plate. Journal of Elasticity 2000; 55; 111-141.

8. Casadei F., Ruzzene M., Dozio L., Cunefare K. A. Broadband vibration control through periodic arrays of resonant shunts: experimental investigation on plates. Smart Materials and Structures 2010; 19(1), 015002.

9. Ciarlet P-G. Mathematical Elasticity, Vol. II: Theory of Plates. North Holland, Amsterdam; 1997.

10. Ciarlet P-G, Destuynder P. A justification of the two-dimensional plate model. Comp. Meth. Appl. Mech. Eng. 1979; 18; 227-258.

11. Collet M., Ouisse M., Ichchou M., Ohayon R. Semi-active optimization of 2D wave dispersion into shunted piezo-composite systems for controlling acoustic interaction. Smart Materials and Structures 2012; 21(9); 094002.

12. Collet M., Ouisse M., Ichchou M. Structural energy flow optimization through adaptive shunted piezoelectric metacomposites. Journal of Intelligent Material Systems and Structures 2012; 23(15); 1661-1677.

13. Figueiredo I.N., Leal C. F. A piezoelectric anisotropic plate model. Asymptotic Analysis 2005; 3-4 ; 327-346. 
14. Ghergu M, Griso G, Mechkour H, Miara B. Homogenization of thin piezoelectric perforated shells. ESAIM, M2AN 2007; 41 (5); 875-895.

15. Lenczner M. Homogénéisation d'un circuit électrique. C.R. Acad. Sci. Paris, série II 1997; 324(9), 537-542.

16. Lenczner M., Senouci-Bereksi, G. Homogenization of electrical networks including voltage-to-voltage amplifiers. Mathematical Models and Methods in Applied Sciences 1999; 9(06), 899-932.

17. Lenczner M., Mercier D. Homogenization of periodic electrical networks including voltage to current amplifiers. Multiscale Modeling \& Simulation 2004; 2(3), 359-397.

18. Licht C, Weller T. Mathematical Modeling of smart material and structures. EastWest J. Math. special volume 2007; 31-49.

19. Nguentseng G. A general convergence result for a functional related to the theory of homogenization. SIAM J. of Math. Anal. 1989; 20 (3); 608-623.

20. Raoult A. Contribution à l'étude des modèles d'évolution de plaques et à l'approximation d'équations d'évolution linéaires du second ordre par des méthodes multipas. Thèse de 3ème cycle, Université Pierre et Marie Curie, Paris, 1980.

21. Ratier N. Analog computing of partial differential equations. IEEE 6th International Conference on in Sciences of Electronics, Technologies of Information and Telecommunications 2012; 275-282.

22. Sanchez-Palencia E. Non-Homogeneous Media and Vibration Theory. Lecture Notes in Physic 127, Springer Verlag; 1980.

23. Sene A. Modelling of piezoelectric static thin plates. Asymptotic Analysis 2001; $127 ; 1-20$.

24. Simon J. Primitives de distributions et applications, Séminaire d'Analyse, Univ. Blaise Pascal 1991 6-7.

25. Thorp O., Ruzzene M., Baz, A. Attenuation and localization of wave propagation in rods with periodic shunted piezoelectric patches. Smart Materials and Structures 2001, 10(5), 979 .

26. Weller T, Licht C. Analyse asymptotique de plaques minces linéairement piézoélectriques. C.R. Acad. Sci. Paris, série I 2002; 335; 309-314.

27. Zemanian A-H. Infinite Electrical Networks. Cambridge University Press, New York; 1991.

28. Zemanian A-H. Transfinite graphs and electrical networks. Trans. Amer. Math. Soc. $1992 ; 334 ; 1-36$. 